\title{
Property-oriented semantics of structured specifications
}

\author{
DONALD SANNELLA ${ }^{\dagger}$ and ANDRZEJ TARLECKI \\ $\dagger$ Laboratory for Foundations of Computer Science, University of Edinburgh, UK \\ Website: homepages.inf.ed.ac.uk/dts/ \\ ¥ Institute of Informatics, University of Warsaw, Poland \\ Website: www.mimuw.edu.pl/ ${ }^{\sim}$ tarlecki/
}

Received 27 July 2012; Revised 6 April 2013

\begin{abstract}
We consider structured specifications built from flat specifications using union, translation and hiding with their standard model-class semantics, in the context of an arbitrary institution. We examine the alternative of sound property-oriented semantics for such specifications, and study their relationship to model-class semantics. An exact correspondence between the two (completeness) is not achievable in general. We show via general results on property-oriented semantics that the semantics arising from the standard proof system is the strongest sound and compositional property-oriented semantics in a wide class of such semantics. We also sharpen one of the conditions that does guarantee completeness and show that it is a necessary condition.
\end{abstract}

\section{Introduction}

Specification formalisms offer specification-building operations for building complex structured specifications by combining and extending simpler ones (Burstall and Goguen, 1977). Then, an understanding of a large specification is achieved via an understanding of its components. The meaning of a specification formalism needs to be completely and precisely defined, and this raises the question of what specifications should denote. The ultimate role of any specification is to describe the class of behaviours that satisfy the specification - its models, in logical terminology - and hence are to be regarded as correct for the task at hand. In algebraic specification, we represent behaviours as algebras, abstracting away from the details of code and algorithms used to implement behaviours. It is then natural to take the class of algebras that represent correct behaviours - its model class - as the semantics of a specification. This view carries over to the framework of an arbitrary logical system formalised as an institution (Goguen and Burstall, 1992), where algebras may be replaced by other semantic structures, as appropriate for modelling behaviours of programs at hand.

However, while model-class semantics remains fundamental, it is vital to be able to determine whether or not a given property is a consequence of a given specification, i.e. holds in all of its models. This is the purpose of proof systems for consequences of structured specifications, as given for instance in (Sannella and Tarlecki, 1988). The essential 
property of such a system is its soundness, which ensures that the consequences derived from a specification do indeed hold in all of its models. Another key property is that the proof system is compositional, so that the consequences of a structured specification are derived from the consequences of its immediate constituents. This allows consequences of structured specifications to be deduced in stages, with the structure of the specification as a guide to the "shape" of the proof. Completeness holds when every property that holds in all of the models of a specification is always derivable; this is highly desirable but is rarely achievable in the practical context of specification formalisms that often provide means for defining the standard model of the natural numbers and other datatypes (MacQueen and Sannella, 1985).

A proof system for consequences of structured specifications determines an alternative "property-oriented" semantics for specifications which maps them to sets of properties (or theories), as in the original semantics of the Clear specification language (Burstall and Goguen, 1980), see also (Diaconescu et al., 1993). Requiring the proof system to be sound amounts to requiring that the properties given by this semantics hold in all of the models given by the model-class semantics. The requirement of compositionality amounts to requiring the meaning of a structured specification in the property-oriented semantics to depend functionally on the meanings of its immediate constituents. Completeness, together with soundness, means that the two forms of semantics essentially coincide.

Sound and compositional property-oriented semantics are the subject of study in this paper, which we conduct in the context of an arbitrary institution (Goguen and Burstall, 1992). We recall the standard property-oriented semantics for structured specifications built from flat specifications using union, translation and hiding, originating from Clear (Burstall and Goguen, 1980), with the model-class semantics given in (Sannella and Tarlecki, 1988). We recall existing results concerning completeness of this semantics and its corresponding proof system, sharpening one of the conditions that guarantee completeness and showing that it is moreover a necessary condition. The semantics is only complete when the logic in use admits interpolation, so for instance there is a "gap" between the model class semantics and the property-oriented semantics for many-sorted equational specifications (unless we impose strong restrictions on the algebras and morphisms involved).

A new result is that the standard property-oriented semantics (and its corresponding proof system) cannot be improved: no sound and compositional semantics can be better. This is a consequence of a more general result we prove, concerning property-oriented semantics for structured specifications built using any collection of specification-building operations. Surprisingly, this result requires a mild but unexpected technical assumption: that the semantics considered must not "forget" any of the axioms present in flat specifications. We first show this under the assumption that an oracle (i.e., a complete proof system) for semantic consequences of any set of axioms in the underlying logic is given and used in the semantics to close the sets of properties assigned to specifications under consequence. Then we show that this assumption may be dropped when a stronger form of compositionality is assumed. Finally, we show how these results carry over to the context of a sound but not necessarily complete proof system for the underlying logic, given as an entailment system. 


\section{Institutional preliminaries}

Following (Goguen and Burstall, 1992) and (Sannella and Tarlecki, 1988), we abstract away from any particular logical system and study specifications built in an arbitrary logical system formalised as an institution.

An institution (Goguen and Burstall, 1992) INS consists of:

- a category Sign $_{\text {INS }}$ of signatures;

- a functor $\operatorname{Sen}_{\text {INS }}: \operatorname{Sign}_{\mathbf{I N S}} \rightarrow$ Set, giving a set $\operatorname{Sen}_{\mathbf{I N S}}(\Sigma)$ of $\Sigma$-sentences for each signature $\Sigma \in\left|\mathbf{S i g n}_{\mathbf{I N S}}\right|$;

- a functor $\operatorname{Mod}_{\text {INS }}: \operatorname{Sign}_{\text {INS }}^{o p} \rightarrow$ Cat, giving a category $\operatorname{Mod}_{\text {INS }}(\Sigma)$ of $\Sigma$-models for each signature $\Sigma \in\left|\mathbf{S i g n}_{\text {INS }}\right|$; and

— a family $\left\langle=_{\mathbf{I N S}, \Sigma} \subseteq\left|\operatorname{Mod}_{\mathbf{I N S}}(\Sigma)\right| \times \operatorname{Sen}_{\mathbf{I N S}}(\Sigma)\right\rangle_{\Sigma \in\left|\operatorname{Sign}_{\text {INS }}\right|}$ of satisfaction relations

such that for any signature morphism $\sigma: \Sigma \rightarrow \Sigma^{\prime}$ the translations $\operatorname{Mod}_{\text {INS }}(\sigma)$ of $\bmod$ els and $\operatorname{Sen}_{\mathbf{I N S}}(\sigma)$ of sentences preserve the satisfaction relation, that is, for any $\varphi \in$ $\operatorname{Sen}_{\mathbf{I N S}}(\Sigma)$ and $M^{\prime} \in\left|\operatorname{Mod}_{\mathbf{I N S}}\left(\Sigma^{\prime}\right)\right|$ the following satisfaction condition holds:

$$
M^{\prime} \models_{\mathbf{I N S}, \Sigma^{\prime}} \operatorname{Sen}_{\mathbf{I N S}}(\sigma)(\varphi) \quad \text { iff } \quad \operatorname{Mod}_{\mathbf{I N S}}(\sigma)\left(M^{\prime}\right) \models_{\mathbf{I N S}, \Sigma} \varphi
$$

Examples of institutions abound. The institution EQ of equational logic has manysorted algebraic signatures as signatures, many-sorted algebras as models and (explicitly quantified) equations as sentences. The institution FOPEQ of first-order predicate logic with equality has signatures that add predicate names to many-sorted algebraic signatures, models that extend algebras by interpreting predicate names as relations, and sentences that are all closed (no free variables) formulae of first-order logic with equality. Then the institution $\mathbf{L}_{\omega_{1} \omega}$ extends FOPEQ by permitting infinitely countable disjunction and conjunction in formulae. See (Sannella and Tarlecki, 2012) for detailed definitions of these and many other institutions. We will also consider single-sorted versions of these institutions $\left(\mathbf{E} \mathbf{Q}^{s s}\right.$ etc.) as well as versions where models are required to have non-empty carriers of all sorts $\left(\mathbf{E Q}^{\text {ne }}\right.$ etc.).

We will freely use standard terminology, and say that a $\Sigma$-model $M$ satisfies a $\Sigma$ sentence $\varphi$, or that $\varphi$ holds in $M$, whenever $M \models_{\mathbf{I N S}, \Sigma} \varphi$. We will omit the subscript INS, writing INS $=\left\langle\right.$ Sign, Sen, Mod, $\left.\left\langle\models_{\Sigma}\right\rangle_{\Sigma \in \mid \text { Sign } \mid}\right\rangle$. Similarly, the subscript $\Sigma$ on the satisfaction relations will often be omitted. For any signature morphism $\sigma: \Sigma \rightarrow \Sigma^{\prime}$, the translation function $\operatorname{Sen}(\sigma): \operatorname{Sen}(\Sigma) \rightarrow \operatorname{Sen}\left(\Sigma^{\prime}\right)$ will be denoted by $\sigma: \operatorname{Sen}(\Sigma) \rightarrow$ $\operatorname{Sen}\left(\Sigma^{\prime}\right)$, the coimage function w.r.t. $\operatorname{Sen}(\sigma)$ by $\sigma^{-1}: \mathcal{P}\left(\operatorname{Sen}\left(\Sigma^{\prime}\right)\right) \rightarrow \mathcal{P}(\operatorname{Sen}(\Sigma))$, and the reduct functor $\operatorname{Mod}(\sigma): \operatorname{Mod}\left(\Sigma^{\prime}\right) \rightarrow \operatorname{Mod}(\Sigma)$ by ${ }_{-}{ }_{\sigma}: \operatorname{Mod}\left(\Sigma^{\prime}\right) \rightarrow \operatorname{Mod}(\Sigma)$. Thus, the satisfaction condition may be re-stated as: $M^{\prime} \models \sigma(\varphi)$ iff $M^{\prime}{ }_{\sigma} \models \varphi$.

From now on we will work with an arbitrary but fixed institution INS $=\left\langle\operatorname{Sign}, \mathbf{S e n}, \mathbf{M o d},\left\langle=_{\Sigma}\right\rangle_{\Sigma \in|\operatorname{Sign}|}\right\rangle$.

For any signature $\Sigma$, the satisfaction relation extends naturally to sets of $\Sigma$-sentences and classes of $\Sigma$-models. Namely, for any set $\Phi \subseteq \operatorname{Sen}(\Sigma)$ of $\Sigma$-sentences and model $M \in|\operatorname{Mod}(\Sigma)|, M \models \Phi$ means $M \models \varphi$ for all $\varphi \in \Phi$. Then, for any $\Sigma$-sentence $\varphi \in \operatorname{Sen}(\Sigma)$ and class $\mathcal{M} \subseteq|\operatorname{Mod}(\Sigma)|$ of $\Sigma$-models, $\mathcal{M} \models \varphi$ means $M \models \varphi$ for all $M \in \mathcal{M}$. Finally, we will also write $\mathcal{M} \models \Phi$ with the obvious meaning.

Given a class of $\Sigma$-models $\mathcal{M} \subseteq|\operatorname{Mod}(\Sigma)|$, its theory is given by $\operatorname{Th}(\mathcal{M})=\{\varphi \in \operatorname{Sen}(\Sigma) \mid$ 
$\mathcal{M} \models \varphi\}$. Given a set of $\Sigma$-sentences $\Phi \subseteq \operatorname{Sen}(\Sigma)$, the class of its models is given by $\operatorname{Mod}(\Phi)=\{M \in|\operatorname{Mod}(\Sigma)||M| \Phi\}$.

For any signature $\Sigma$, a $\Sigma$-sentence $\varphi \in \operatorname{Sen}(\Sigma)$ is a semantic consequence of a set of $\Sigma$-sentences $\Phi \subseteq \operatorname{Sen}(\Sigma)$, written $\Phi \models_{\Sigma} \varphi$ or simply $\Phi \models \varphi$, if for all $\Sigma$-models $M \in|\operatorname{Mod}(\Sigma)|, M \models \varphi$ whenever $M \models \Phi$.

It is trivial to check that for any class of $\Sigma$-models $\mathcal{M} \subseteq|\operatorname{Mod}(\Sigma)|$, its theory $T h(\mathcal{M})$ is closed under semantic consequence, and that if a set $\Phi \subseteq \operatorname{Sen}(\Sigma)$ is closed under semantic consequence $(\Phi \models \varphi$ implies $\varphi \in \Phi)$ then it is a theory of its model class. We write $C l_{\Sigma}(\Phi)$ for the closure of $\Phi$ under semantic consequence, $C l_{\Sigma}(\Phi)=\operatorname{Th}(\operatorname{Mod}(\Phi))$.

Translation under signature morphisms preserves semantic consequence: for any $\sigma: \Sigma \rightarrow$ $\Sigma^{\prime}, \varphi \in \operatorname{Sen}(\Sigma)$ and $\Phi \subseteq \operatorname{Sen}(\Sigma)$, if $\Phi=\varphi$ then $\sigma(\Phi) \models \sigma(\varphi)$. The opposite implication may fail in general. However, it holds for instance if the reduct $\left.{ }_{-}\right|_{\sigma}: \operatorname{Mod}\left(\Sigma^{\prime}\right) \rightarrow \operatorname{Mod}(\Sigma)$ is surjective on models, and so $\Phi \models \varphi$ iff $\sigma(\Phi) \models \sigma(\varphi)$ then. Consequently, semantic consequence is (preserved and) reflected by translation under all signature morphisms that are injective in $\mathbf{E} \mathbf{Q}^{n e}, \mathbf{F O P E Q} \mathbf{Q}^{n e}$ and $\mathbf{L}_{\omega_{1} \omega}^{n e}$ (since in these institutions injective morphisms induce surjective reduct functors).

Institutional structure is rich enough to enable a number of key features of logical systems to be expressed. For instance, amalgamation and interpolation properties may be captured as follows.

Consider the following commuting diagram in Sign:

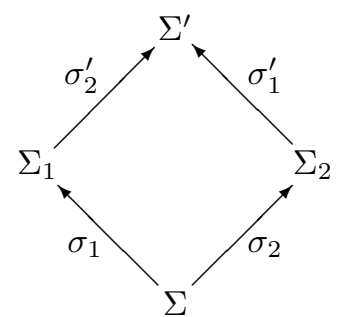

This diagram admits amalgamation if for any two models $M_{1} \in\left|\operatorname{Mod}\left(\Sigma_{1}\right)\right|$ and $M_{2} \in$ $\left|\operatorname{Mod}\left(\Sigma_{2}\right)\right|$ such that $\left.M_{1}\right|_{\sigma_{1}}=\left.M_{2}\right|_{\sigma_{2}}$, there exists a unique model $M^{\prime} \in\left|\operatorname{Mod}\left(\Sigma^{\prime}\right)\right|$ such that $\left.M^{\prime}\right|_{\sigma_{2}^{\prime}}=M_{1}$ and $\left.M^{\prime}\right|_{\sigma_{1}^{\prime}}=M_{2}$ (we call such $M^{\prime}$ the amalgamation of $M_{1}$ and $M_{2}$ ), and similarly for model morphisms.

An institution is semi-exact if pushouts of signature morphisms always exist and admit amalgamation (or equivalently, Mod: $\mathbf{S i g n}^{o p} \rightarrow$ Cat translates them to pullbacks in Cat). In fact, the developments below do not depend on the amalgamation properties for model morphisms, so semi-exactness is a bit too strong for our needs. We use this standard notion nonetheless, since we are not aware of any example of an institution of practical importance where pushout diagrams admit amalgamation of models but not of morphisms.

Another way to weaken the requirement of exactness is to drop uniqueness of the amalgamation; in fact, the results below still hold if we require institutions to be weakly semi-exact, i.e. map the pushouts considered in the category of signatures to weak pullbacks in Cat. Again, we refrain from this possible generalisation since amalgamation, rather than weak amalgamation, is a crucial property of "useful" logical systems that 
enables systematic combination of models (that represent programs) in architectural designs (Sannella and Tarlecki, 2012).

It is well-known that $\mathbf{E Q}, \mathbf{F O P E Q}$ and $\mathbf{L}_{\omega_{1} \omega}$ (also their single-sorted versions) are semi-exact. But this fails for some other institutions of interest, where it is useful to rely on a slightly more subtle notion, parameterised by additional classes of signature morphisms.

Consider two classes $\mathcal{H}, \mathcal{W} \subseteq|\mathbf{S i g n}|$ of signature morphisms. ${ }^{1}$ INS is $\langle\mathcal{H}, \mathcal{W}\rangle$-exact if for any signature morphisms $\delta \in \mathcal{H}$ and $\tau \in \mathcal{W}$ with a common source there are $\delta^{\prime} \in \mathcal{H}$ and $\tau^{\prime} \in \mathcal{W}$ forming a pushout

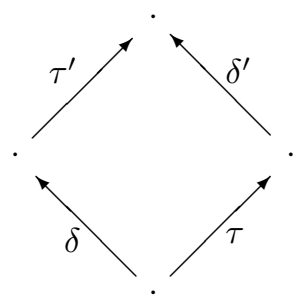

that admits amalgamation; then any such pushout admits amalgamation as well.

In the following we will always assume that $\mathcal{H}$ and $\mathcal{W}$ form wide subcategories of Sign (i.e., are closed under composition and contain all identities in Sign) and that $\mathcal{H} \subseteq \mathcal{W}$.

Consider again the following commuting diagram in Sign:

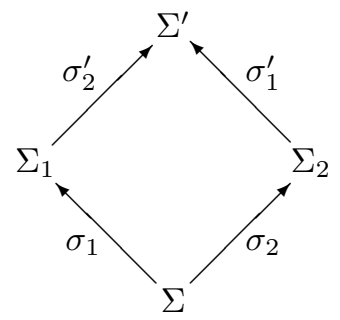

The above diagram admits parameterised (or Craig-Robinson) interpolation if for any $\Phi_{1} \subseteq \operatorname{Sen}\left(\Sigma_{1}\right), \Phi_{2} \subseteq \operatorname{Sen}\left(\Sigma_{2}\right)$ and $\varphi_{2} \in \operatorname{Sen}\left(\Sigma_{2}\right)$, whenever $\sigma_{2}^{\prime}\left(\Phi_{1}\right) \cup \sigma_{1}^{\prime}\left(\Phi_{2}\right) \models \sigma_{1}^{\prime}\left(\varphi_{2}\right)$ then for some $\Phi \subseteq \operatorname{Sen}(\Sigma)$ such that $\Phi_{1} \models \sigma_{1}(\Phi)$ we have $\Phi_{2} \cup \sigma_{2}(\Phi) \models \varphi_{2}$. Such a set $\Phi$ will be called a set of interpolants for $\Phi_{1}$ and $\varphi_{2}$ w.r.t. $\Phi_{2}$. The diagram admits Craig interpolation if it admits parameterised interpolation with "parameter set" $\Phi_{2}=\emptyset$.

Given classes $\mathcal{H}, \mathcal{W} \subseteq$ Sign of signature morphisms, we say that INS admits parameterised (resp. Craig) $\langle\mathcal{H}, \mathcal{W}\rangle$-interpolation if for any signature morphisms $\delta \in \mathcal{H}$ and $\tau \in \mathcal{W}$ with a common source there are $\delta^{\prime} \in \mathcal{H}$ and $\tau^{\prime} \in \mathcal{W}$ forming a pushout

\footnotetext{
${ }^{1}$ In the context of structured specifications - see Sect. 3 - morphisms in $\mathcal{H}$ will be used for hide (hiding) while those in $\mathcal{W}$ will be used for with (translation).
} 


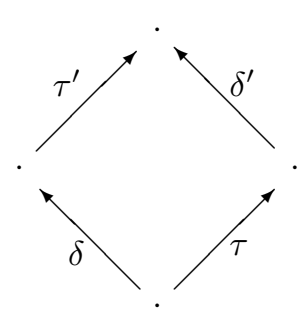

that admits parameterised (resp. Craig) interpolation; then any such pushout admits parameterised (resp. Craig) interpolation as well.

The above reformulation of classical (first-order) Craig interpolation (Chang and Keisler, 1990) has its source in (Tarlecki, 1986). We resign the requirement that the interpolant be given by a single formula, following what is more natural for instance for equational logic, as argued in (Rodenburg, 1991) and (Diaconescu et al., 1993). It is well-known that single-sorted first-order predicate logic with equality, $\mathbf{F O P E Q ^ { s s }}$, admits Craig as well as parameterised interpolation. But in the many-sorted case, interpolation requires additional assumptions on the signature morphisms involved: FOPEQ admits Craig and parameterised $\langle\mathcal{H}, \mathcal{W}\rangle$-interpolation when all morphisms in $\mathcal{H}$ are injective on sorts, see (Borzyszkowski, 2005).

Interpolation properties for equational logic are a bit more delicate. $\mathbf{E Q}^{\text {ne }}$ admits Craig $\langle\mathcal{H}, \mathcal{W}\rangle$-interpolation for classes $\mathcal{H}$ and $\mathcal{W}$ where all morphisms are injective, but the restriction to non-empty carriers cannot be dropped (Roşu and Goguen, 2000), (Tarlecki, 2011). Parameterised $\langle\mathcal{H}, \mathcal{W}\rangle$-interpolation for $\mathbf{E Q}^{\text {ne }}$ fails - a counterexample may be extracted from Example 4.3 below, cf. Prop. 4.5 - unless injectivity and strong "encapsulation" properties are imposed on the morphisms in $\mathcal{H}$ (Diaconescu, 2008).

In the framework of first-order predicate logic, it is easy to derive the (stronger) parameterised interpolation property from Craig interpolation. This relies on compactness and closure of the set of first-order sentences under conjunction and implication as follows. Consider the following commuting diagram in the category of first-order signatures

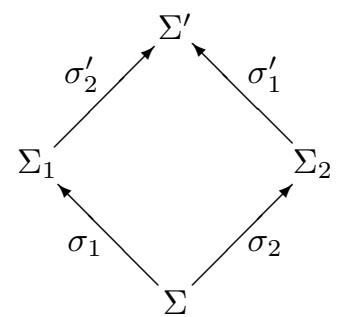

and assume that it admits Craig interpolation. Let $\Phi_{1} \subseteq \operatorname{Sen}\left(\Sigma_{1}\right), \Phi_{2} \subseteq \operatorname{Sen}\left(\Sigma_{2}\right)$ and $\varphi \in$ $\operatorname{Sen}\left(\Sigma_{2}\right)$ be such that $\sigma_{2}^{\prime}\left(\Phi_{1}\right) \cup \sigma_{1}^{\prime}\left(\Phi_{2}\right) \models \sigma_{1}^{\prime}(\varphi)$. By compactness, there are finite $\Psi_{1} \subseteq$ $\Phi_{1}$ and $\Psi_{2} \subseteq \Phi_{2}$ such that $\sigma_{2}^{\prime}\left(\Psi_{1}\right) \cup \sigma_{1}^{\prime}\left(\Psi_{2}\right) \models \sigma_{1}^{\prime}(\varphi)$. Then $\sigma_{2}^{\prime}\left(\Psi_{1}\right) \models \sigma_{1}^{\prime}\left(\bigwedge \Psi_{2} \Rightarrow \varphi\right)$, and so by the simple Craig interpolation property, we have a set $\Psi$ of $\Sigma$-sentences such that $\Psi_{1} \models \sigma_{1}(\Psi)$ and $\sigma_{2}(\Psi) \models\left(\bigwedge \Psi_{2} \Rightarrow \varphi\right)$. Then also $\Phi_{1} \models \sigma_{1}(\Psi)$ and $\Phi_{2} \cup \sigma_{2}(\Psi) \models \varphi$, so $\Psi$ is an interpolant set for $\Phi_{1}$ and $\varphi$ w.r.t. $\Phi_{2}$. Although this argument may be generalised to any institution where implication and "sufficiently large" conjunction are expressible, in general parameterised interpolation is properly stronger than Craig interpolation.

Just as in classical first-order logic, where interpolation is sometimes derived from the 
Robinson consistency theorem, or from various conservativity properties, similar relationships hold between analogous notions in the institutional framework. For instance, we say that $\sigma_{1}: \Sigma \rightarrow \Sigma_{1}$ is conservative for $\Phi_{1} \subseteq \operatorname{Sen}\left(\Sigma_{1}\right)^{2}$ if $\operatorname{Mod}\left(\sigma_{1}^{-1}\left(C l_{\Sigma_{1}}\left(\Phi_{1}\right)\right)\right)=$ $\left.\operatorname{Mod}\left(\Phi_{1}\right)\right|_{\sigma_{1}}$, i.e., every model of the $\sigma_{1}$-coimage of the theory generated by $\Phi_{1}$ has a $\sigma_{1}$-expansion that satisfies $\Phi_{1}$. Given a pushout as above, and $\Phi_{1} \subseteq \operatorname{Sen}\left(\Sigma_{1}\right), \Phi_{2} \subseteq$ $\operatorname{Sen}\left(\Sigma_{2}\right), \varphi_{2} \in \operatorname{Sen}\left(\Sigma_{2}\right)$ such that $\sigma_{2}^{\prime}\left(\Phi_{1}\right) \cup \sigma_{1}^{\prime}\left(\Phi_{2}\right) \models \sigma_{1}^{\prime}\left(\varphi_{2}\right)$, it is easy to check that $\sigma_{1}^{-1}\left(C l_{\Sigma_{1}}\left(\Phi_{1}\right)\right)$ is a set of interpolants for $\Phi_{1}$ and $\varphi_{2}$ w.r.t. $\Phi_{2}$ whenever $\sigma_{1}: \Sigma \rightarrow \Sigma_{1}$ is conservative for $\Phi_{1}$. So, conservativity in this sense is a stronger property than parameterised interpolation; in fact, easy examples show that it is strictly stronger.

\section{Structured specifications}

As announced in Sect. 2, we will work with an arbitrary institution $\mathbf{I N S}=\left\langle\operatorname{Sign}, \mathbf{S e n}, \mathbf{M o d},\left\langle\models_{\Sigma}\right\rangle_{\Sigma \in|\operatorname{Sign}|}\right\rangle$ equipped with two classes $\mathcal{H} \subseteq \mathcal{W} \subseteq$ Sign of signature morphisms that contain all identities and are closed under composition.

We study specifications built in INS. Whatever exactly the specifications are, and however exactly they are written, each specification has to determine a class of programs that correctly realise it. If the models of the institution capture (the semantics of) the programs we want to deal with, with signatures capturing their (static) interfaces, then the most basic semantics of a specification is given in terms of its signature and its class of models. Consequently, we will consider a class Spec of specifications in INS with a semantics that for each specification $S P \in$ Spec defines its signature $\operatorname{Sig}[S P]$ and its class of models $\operatorname{Mod}[S P] \subseteq|\operatorname{Mod}(\operatorname{Sig}[S P])|$. We will refer to specifications $S P$ with $\operatorname{Sig}[S P]=$ $\Sigma$ as $\Sigma$-specifications and write $\operatorname{Spec}(\Sigma)$ for the class of all $\Sigma$-specifications. When we want to stress that we are dealing with specifications built in the particular institution INS, we will write $\operatorname{Spec}_{\text {INS }}$ and $\operatorname{Spec}_{\mathbf{I N S}}(\Sigma)$ rather than just $\operatorname{Spec}$ and $\operatorname{Spec}(\Sigma)$, respectively.

The semantics determines an obvious notion of specification equivalence: specifications $S P_{1}$ and $S P_{2}$ are equivalent, written $S P_{1} \equiv S P_{2}$, if their semantics coincide: $\operatorname{Sig}\left[S P_{1}\right]=$ $\operatorname{Sig}\left[S P_{2}\right]$ and $\operatorname{Mod}\left[S P_{1}\right]=\operatorname{Mod}\left[S P_{2}\right]$.

The simplest specifications are presentations which simply give a set of axioms asserting the required properties. We write such flat specifications as $\langle\Sigma, \Phi\rangle$ for any $\Sigma \in \mid$ Sign| and $\Phi \subseteq \operatorname{Sen}(\Sigma)$ and define their semantics in the obvious way:

$$
\begin{aligned}
\operatorname{Sig}[\langle\Sigma, \Phi\rangle] & =\Sigma \\
\operatorname{Mod}[\langle\Sigma, \Phi\rangle] & =\{M \in|\operatorname{Mod}(\Sigma)| \mid M \models \Phi\}
\end{aligned}
$$

Following the ideas of (Burstall and Goguen, 1977) and (Burstall and Goguen, 1981), more complex specifications are now systematically formed using a collection of specificationbuilding operations. This stratified way of designing specification formalisms, with a clear separation of basic blocks given as flat specifications from specification structuring mechanisms which are largely independent of the underlying logic, is by now standard and

\footnotetext{
2 That is, stretching the terminology of (Goguen and Roşu, 2004) somewhat, the module $\left\langle\sigma_{1}: \Sigma \rightarrow \Sigma_{1}, \Phi_{1}\right\rangle$ is conservative
} 
can be usefully exploited to ensure clarity and reusability in the context of different logical systems formalised as institutions. One example of a specification formalism that is structured in this way is CASL (Bidoit and Mosses, 2004; Mosses, 2004).

We will presume that specification-building operations are "strongly typed" by specification signatures, and write

$$
\text { sbo: } \operatorname{Spec}\left(\Sigma_{1}\right) \times \ldots \times \operatorname{Spec}\left(\Sigma_{n}\right) \rightarrow \operatorname{Spec}(\Sigma)
$$

to indicate that a specification-building operation sbo takes any specifications $S P_{1} \in$ $\operatorname{Spec}\left(\Sigma_{1}\right), \ldots, S P_{n} \in \operatorname{Spec}\left(\Sigma_{n}\right)$ and yields a specification $\operatorname{sbo}\left(S P_{1}, \ldots, S P_{n}\right) \in \operatorname{Spec}(\Sigma)$. The meaning of such a specification-building operation is then given as a function on classes of models:

$$
\llbracket \mathbf{s b o} \rrbracket: \mathcal{P}\left(\left|\operatorname{Mod}\left(\Sigma_{1}\right)\right|\right) \times \ldots \times \mathcal{P}\left(\left|\operatorname{Mod}\left(\Sigma_{n}\right)\right|\right) \rightarrow \mathcal{P}(|\operatorname{Mod}(\Sigma)|)
$$

The semantics of specifications is then given compositionally, by defining the model class of $\mathbf{s b o}\left(S P_{1}, \ldots, S P_{n}\right)$ in terms of the model classes of $S P_{1}, \ldots, S P_{n}$ using $\llbracket \mathbf{s b o} \rrbracket$.

Flat specifications may be viewed as constant (nullary) specification-building operations. In addition to flat specifications, we will concentrate here on three (families of) kernel specification-building operations, originating from ASL (Sannella and Wirsing, 1983), and then re-introduced in (Sannella and Tarlecki, 1988) with institution-independent model-class semantics. These operations are also at the core of CASL, and we will use here a notation closer to the syntax of CASL, see also (Sannella and Tarlecki, 2012).

Union: For any signature $\Sigma$, we have $\cup_{-}: \operatorname{Spec}(\Sigma) \times \operatorname{Spec}(\Sigma) \rightarrow \operatorname{Spec}(\Sigma)$ with $\llbracket-\cup_{-} \rrbracket=$ $\left(\cap_{-}\right)$. That is, given $\Sigma$-specifications $S P_{1}$ and $S P_{2}, S P_{1} \cup S P_{2}$ is a specification with the following semantics:

$$
\begin{aligned}
\operatorname{Sig}\left[S P_{1} \cup S P_{2}\right] & =\Sigma \\
\operatorname{Mod}\left[S P_{1} \cup S P_{2}\right] & =\operatorname{Mod}\left[S P_{1}\right] \cap \operatorname{Mod}\left[S P_{2}\right]
\end{aligned}
$$

$S P_{1} \cup S P_{2}$ combines the constraints imposed by $S P_{1}$ and $S P_{2}$.

Translation: For any signature morphism $\sigma: \Sigma \rightarrow \Sigma^{\prime}$ in $\mathcal{W}$, we have _ with $\sigma: \operatorname{Spec}(\Sigma) \rightarrow$

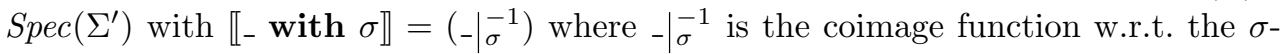
reduct of models. That is, given any $\Sigma$-specification $S P, S P$ with $\sigma$ is a specification with the following semantics:

$$
\begin{aligned}
\operatorname{Sig}[S P \text { with } \sigma] & =\Sigma^{\prime} \\
\operatorname{Mod}[S P \text { with } \sigma] & =\left\{M^{\prime} \in\left|\operatorname{Mod}\left(\Sigma^{\prime}\right)\right|\left|M^{\prime}\right|_{\sigma} \in \operatorname{Mod}[S P]\right\}
\end{aligned}
$$

$S P$ with $\sigma$ changes the names in $S P$ according to $\sigma$, also adding new components.

Hiding: For any signature morphism $\sigma: \Sigma^{\prime} \rightarrow \Sigma$ in $\mathcal{H}$, we have _ hide via $\sigma: \operatorname{Spec}(\Sigma) \rightarrow$

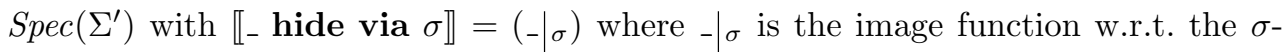
reduct of models. That is, given any $\Sigma$-specification $S P, S P$ hide via $\sigma$ is a specification with the following semantics:

$$
\begin{aligned}
\operatorname{Sig}[S P \text { hide via } \sigma] & =\Sigma^{\prime} \\
\operatorname{Mod}[S P \text { hide via } \sigma] & =\{M|\sigma| M \in \operatorname{Mod}[S P]\}
\end{aligned}
$$

$S P$ hide via $\sigma$ views $S P$ as a $\Sigma^{\prime}$-specification, hiding auxiliary components.

We will write $S p e c^{U T H}$ for the class of specifications built from flat specifications using union of specifications over common signatures, translation along morphisms in $\mathcal{W}$, and hiding w.r.t. morphisms in $\mathcal{H}$. Note that the definitions of the syntax and of the signature 
for specifications in $S p e c^{U T H}$ do not depend on the models and satisfaction relations of the institution involved (although they are used to determine the model-class semantics of specifications, of course) but only on the category of signatures Sign, with indicated classes $\mathcal{H}, \mathcal{W} \subseteq$ Sign, and sentences given by the functor Sen: Sign $\rightarrow$ Set. When we want to make this dependency and the independence from the other components of the institution more explicit, we write $\operatorname{Spec}_{\text {Sen }}^{U T H}$ for $S p e c^{U T H}$. However, in general there may be specification-building operations that involve the model part (or satisfaction relations) of the underlying institution even in the formulation of "syntax" - see for instance the singleton operation used in (Sannella et al., 1992).

A specification is finitary if all the flat specifications it involves have a finite set of axioms.

The following normal form theorem provides an important technical tool:

Theorem 3.1. If INS is $\langle\mathcal{H}, \mathcal{W}\rangle$-exact then any specification $S P \in S_{\text {Pec }}{ }^{U T H}$ has an equivalent normal form $\operatorname{nf}(S P)$ given as $\left\langle\Sigma^{\prime}, \Phi^{\prime}\right\rangle$ hide via $\sigma$, for some $\Sigma^{\prime} \in|\operatorname{Sign}|$, $\sigma: \operatorname{Sig}[S P] \rightarrow \Sigma^{\prime}$ in $\mathcal{H}$, and $\Phi^{\prime} \subseteq \operatorname{Sen}\left(\Sigma^{\prime}\right)$. Moreover, $\Phi^{\prime}$ is finite if $S P$ is finitary.

We omit the explicit inductive definition of $\operatorname{nf}(S P)$ and the proof of equivalence - such normal form results are well-known since (Bergstra et al., 1990), with predecessor in (Ehrig et al., 1983) and the current general version in (Borzyszkowski, 2002). Let us just mention that (weak) $\langle\mathcal{H}, \mathcal{W}\rangle$-exactness of the institution considered is crucial here.

In (Goguen and Roşu, 2004), specifications of the form $\langle\Sigma, \Phi\rangle$ hide via $\sigma$ are taken as the basic meanings of specification expressions. The above theorem shows that this brings no loss with respect to the model-class semantics, at least for specifications built using the operations introduced above.

\section{Property-oriented semantics for structured specifications}

While we view the semantics of specifications given in terms of their model classes as the most basic, their logical consequences are obviously of prime importance.

A $\Sigma$-sentence $\varphi \in \operatorname{Sen}(\Sigma)$ is a semantic consequence of a $\Sigma$-specification $S P \in \operatorname{Spec}(\Sigma)$ if $\operatorname{Mod}[S P] \models \varphi$; we write this $S P \models \varphi$. The set of all semantic consequences of $S P$, called the theory of $S P$, is denoted by $T h(S P)$, so $T h(S P)=T h(\operatorname{Mod}[S P])$, and in particular $\operatorname{Th}(\langle\Sigma, \Phi\rangle)=\operatorname{Th}(\operatorname{Mod}[\langle\Sigma, \Phi\rangle])=C l_{\Sigma}(\Phi)$.

Some authors go as far as to take the theory assigned to a specification as its meaning. This goes back to Clear (Burstall and Goguen, 1980), and is the stance taken in (Diaconescu et al., 1993). In this section and the next we will discuss this option and its relationship with the model-class semantics defined above.

By a property-oriented semantics for specifications we mean any function $\mathcal{T}$ that assigns to each specification $S P \in S p e c$ a set $\mathcal{T}(S P) \subseteq \operatorname{Sen}(\operatorname{Sig}[S P])$ of $\operatorname{Sig}[S P]$-sentences.

The assignment $T h$ that maps each specification $S P$ to its theory $T h(S P)$ is one such semantics. In fact, this is the "best" such semantics in the sense that it captures all and only the properties that hold in all models of the given specification. We will use it as a yardstick to measure the "strength" and "soundness" of other such semantics. 
Here is some vocabulary to talk about properties of such semantics. Let $\mathcal{T}$ be a property-oriented semantics for specifications. Then:

- $\mathcal{T}$ is sound if $\mathcal{T}(S P) \subseteq T h(S P)$ for every specification $S P \in$ Spec.

- A sound $\mathcal{T}$ is complete if $\mathcal{T}(S P)=T h(S P)$ for every specification $S P \in$ Spec.

- $\mathcal{T}$ is monotone for a specification-building operation sbo: $\operatorname{Spec}\left(\Sigma_{1}\right) \times \ldots \times \operatorname{Spec}\left(\Sigma_{n}\right) \rightarrow$ $\operatorname{Spec}(\Sigma)$ if $\mathcal{T}\left(\operatorname{sbo}\left(S P_{1}, \ldots, S P_{n}\right)\right) \subseteq \mathcal{T}\left(\mathbf{s b o}\left(S P_{1}^{\prime}, \ldots, S P_{n}^{\prime}\right)\right)$ for all specifications $S P_{1}, S P_{1}^{\prime} \in$ $\operatorname{Spec}\left(\Sigma_{1}\right), \ldots, S P_{n}^{\prime}, S P_{n}^{\prime} \in \operatorname{Spec}\left(\Sigma_{n}\right)$ such that $\mathcal{T}\left(S P_{i}\right) \subseteq \mathcal{T}\left(S P_{i}^{\prime}\right)$, for $i=1, \ldots, n$.

- $\mathcal{T}$ is compositional for a specification-building operation sbo: $\operatorname{Spec}\left(\Sigma_{1}\right) \times \ldots \times \operatorname{Spec}\left(\Sigma_{n}\right) \rightarrow$ $\operatorname{Spec}(\Sigma)$ if $\mathcal{T}\left(\operatorname{sbo}\left(S P_{1}, \ldots, S P_{n}\right)\right)=\mathcal{T}\left(\mathbf{s b o}\left(S P_{1}^{\prime}, \ldots, S P_{n}^{\prime}\right)\right)$ for all specifications $S P_{1}, S P_{1}^{\prime} \in$ $\operatorname{Spec}\left(\Sigma_{1}\right), \ldots, S P_{n}^{\prime}, S P_{n}^{\prime} \in \operatorname{Spec}\left(\Sigma_{n}\right)$ such that $\mathcal{T}\left(S P_{i}\right)=\mathcal{T}\left(S P_{i}^{\prime}\right)$, for $i=1, \ldots, n$.

- A sound $\mathcal{T}$ is closed-complete for a specification-building operation sbo: $\operatorname{Spec}\left(\Sigma_{1}\right) \times$ $\ldots \times \operatorname{Spec}\left(\Sigma_{n}\right) \rightarrow \operatorname{Spec}(\Sigma)$ if $\mathcal{T}\left(\operatorname{sbo}\left(S P_{1}, \ldots, S P_{n}\right)\right)=\operatorname{Th}\left(\mathbf{s b o}\left(S P_{1}, \ldots, S P_{n}\right)\right)$ for all $S P_{1} \in \operatorname{Spec}\left(\Sigma_{1}\right), \ldots, S P_{n} \in \operatorname{Spec}\left(\Sigma_{n}\right)$ such that $\operatorname{Mod}_{S i g\left[S P_{i}\right]}\left(\mathcal{T}\left(S P_{i}\right)\right)=\operatorname{Mod}\left[S P_{i}\right]$, $i=1, \ldots, n$.

- $\mathcal{T}$ is flat-complete if $\mathcal{T}(\langle\Sigma, \Phi\rangle)=C l_{\Sigma}(\Phi)$ for every signature $\Sigma$ and set $\Phi$ of $\Sigma$ sentences.

- $\mathcal{T}$ is extensive if $\Phi \subseteq \mathcal{T}(\langle\Sigma, \Phi\rangle)$ for every signature $\Sigma$ and set $\Phi$ of $\Sigma$-sentences.

- $\mathcal{T}$ is theory-oriented if for all specifications $S P \in S p e c, \mathcal{T}(S P)$ is a theory (i.e., a set of sentences that is closed under semantic consequence).

$\mathcal{T}$ is monotone (resp. compositional, closed-complete) if it is so for all specificationbuilding operations in use.

Soundness is the property we must insist on for any property-oriented semantics. Completeness is the goal we should aim to soundly approximate as accurately as possible. Compositionality (implied by monotonicity) is a crucial property needed to deal with large structured specifications in a modular way. Closed-completeness is a technical notion to capture how accurate the semantics is for a given specification-building operation: we want the semantics for a specification built using an operation to be complete at least under the assumption that it exactly captures the model classes of the argument specifications (which for theory-oriented semantics is properly stronger than completeness of the semantics for the argument specifications). Flat-completeness is closed-completeness for flat specifications as nullary specification-building operations. Extensiveness requires the semantics of a flat specification to include all of its axioms. Surprisingly, this simple technical condition turns out to play a key role in the results below. Clearly, any flat-complete semantics is extensive. We usually expect semantics to be theory-oriented: we could in principle always close the set of properties given in one way or another under semantic consequences. But this would make our analysis of the issues of dealing with structured specifications more restrictive, and potentially dependent on the completeness of entailment used for the underlying logical system. Any (flat-complete and) closed-complete semantics is theory-oriented. Any extensive and theory-oriented semantics is flat-complete.

The semantics Th above, defined via the model-class semantics for specifications, is sound, complete and theory-oriented. It is compositional for hiding: for any signature morphism $\sigma: \Sigma^{\prime} \rightarrow \Sigma$ and $\Sigma$-specification $S P$, Th $(S P$ hide via $\sigma)=\sigma^{-1}(T h(S P))$ (San- 
nella and Tarlecki, 1988); note that $\sigma^{-1}(\Phi)$ is a theory if $\Phi$ is a theory, by the satisfaction condition.

A key drawback of $T h$ is that it is not compositional for union and translation. Here are counterexamples to illustrate this. We present these by constructing an artificial institution in which the point is clear, but the reader is encouraged to look for analogous situations in more standard logical systems.

Example 4.1. Consider an institution INS with exactly two signatures $\Sigma$ and $\Sigma^{\prime}$, and $\sigma: \Sigma \rightarrow \Sigma^{\prime}$ as the only non-identity signature morphism. Let $\operatorname{Sen}(\Sigma)=\left\{\varphi, \varphi^{\prime}\right\}$, $\operatorname{Sen}\left(\Sigma^{\prime}\right)=\left\{\varphi, \varphi^{\prime}, \psi_{1}, \psi_{2}\right\}$, with $\sigma$-translation preserving $\varphi$ and $\varphi^{\prime}$, and let $|\operatorname{Mod}(\Sigma)|=$ $\left|\operatorname{Mod}\left(\Sigma^{\prime}\right)\right|=\left\{M_{1}, M_{2}\right\}$, with the identity $\sigma$-reduct. Define $M_{1} \models_{\Sigma} \varphi, M_{2} \models_{\Sigma} \varphi$, $M_{1} \not \nvdash_{\Sigma} \varphi^{\prime}, M_{2} \not \models_{\Sigma} \varphi^{\prime}$, and $M_{1} \models_{\Sigma^{\prime}} \varphi, M_{2} \models_{\Sigma^{\prime}} \varphi, M_{1} \not \nvdash_{\Sigma^{\prime}} \varphi^{\prime}, M_{2} \not \nvdash_{\Sigma^{\prime}} \varphi^{\prime}, M_{1} \models_{\Sigma^{\prime}} \psi_{1}$, $M_{2} \not \nvdash_{\Sigma^{\prime}} \psi_{1}, M_{1} \not \nvdash_{\Sigma^{\prime}} \psi_{2}, M_{2}=_{\Sigma^{\prime}} \psi_{2}$.

$\operatorname{In} \Sigma^{\prime}$, we have $\operatorname{Mod}\left(\left\{\psi_{1}\right\}\right)=\left\{M_{1}\right\}$ and $\operatorname{Mod}\left(\left\{\psi_{2}\right\}\right)=\left\{M_{2}\right\}$. Let $S P_{1}$ be $\left\langle\Sigma^{\prime},\left\{\psi_{1}\right\}\right\rangle$ hide via $\sigma$ and $S P_{2}$ be $\left\langle\Sigma^{\prime},\left\{\psi_{2}\right\}\right\rangle$ hide via $\sigma$. Then $\operatorname{Mod}\left[S P_{1}\right]=\left\{M_{1}\right\}$ and $\operatorname{Mod}\left[S P_{2}\right]=\left\{M_{2}\right\}$, yielding $\operatorname{Th}\left(S P_{1}\right)=\{\varphi\}=\operatorname{Th}\left(S P_{2}\right)$. Now:

$-\operatorname{Mod}\left[S P_{1} \cup S P_{2}\right]=\emptyset$ so $T h\left(S P_{1} \cup S P_{2}\right)=\left\{\varphi, \varphi^{\prime}\right\}$, which is distinct from $\operatorname{Th}\left(S P_{2} \cup\right.$ $\left.S P_{2}\right)=\operatorname{Th}\left(S P_{2}\right)=\{\varphi\}$.

- $\operatorname{Th}\left(S P_{1}\right.$ with $\left.\sigma\right)=\left\{\varphi, \psi_{1}\right\}$, which is distinct from $\operatorname{Th}\left(S P_{2}\right.$ with $\left.\sigma\right)=\left\{\varphi, \psi_{2}\right\}$.

This shows that $T h$ is compositional for neither union nor translation.

The lack of compositionality of Th for union and translation, as well as natural consideration of proof-theoretic issues (see Sect. 7 below), led to the following standard compositional property-oriented semantics $\mathcal{T}_{\text {INS }}$ for specifications in $\operatorname{Spec}_{\text {INS }}^{U T H}$. This semantics originates from the proof rules in (Sannella and Tarlecki, 1988), was given in (Bergstra et al., 1990) and was used in (Diaconescu et al., 1993). Here is the inductive definition:

$$
\begin{aligned}
& \mathcal{T}_{\text {INS }}(\langle\Sigma, \Phi\rangle)=C l_{\Sigma}(\Phi) \\
& \mathcal{T}_{\text {INS }}\left(S P \cup S P^{\prime}\right)=C l_{S i g[S P]}\left(\mathcal{T}_{\text {INS }}(S P) \cup \mathcal{T}_{\text {INS }}\left(S P^{\prime}\right)\right) \\
& \mathcal{T}_{\text {INS }}(S P \text { with } \sigma: S i g[S P] \rightarrow \Sigma)=C l_{\Sigma}\left(\sigma\left(\mathcal{T}_{\text {INS }}(S P)\right)\right) \\
& \mathcal{T}_{\text {INS }}(S P \text { hide via } \sigma: \Sigma \rightarrow \operatorname{Sig}[S P])=\sigma^{-1}\left(\mathcal{T}_{\text {INS }}(S P)\right)
\end{aligned}
$$

Proposition 4.2. $\mathcal{T}_{\text {INS }}$ is a sound theory-oriented semantics for specifications built from flat specifications using union, translation and hiding. It is monotone, compositional, extensive, flat-complete and closed-complete for union, translation and hiding.

Proof. Monotonicity and compositionality follow from the definitions, while soundness requires a simple inductive proof. Closed-completeness for hiding follows from the definitions and the satisfaction condition. (Soundness, monotonicity and closed-completeness were shown in (Sannella and Tarlecki, 1988).)

The missing property is completeness - and indeed $\mathcal{T}_{\text {INS }}$ is not complete, as the following counterexample shows. 
Example 4.3. Consider the following specifications built in the institution EQ of equational logic, using a hopefully self-explanatory notation based on the syntax of $\mathrm{CASL}^{3}$ :

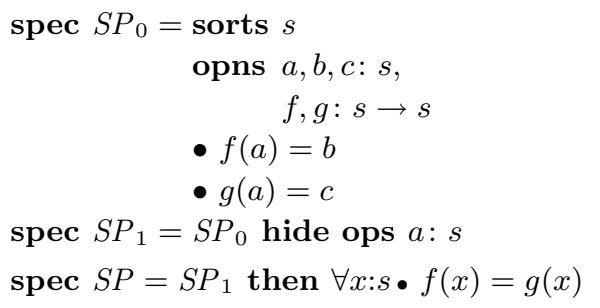

This example relies on the fact that the class of models of any set of equations is closed under subalgebras. Note that using conditional equations would not help, as this property holds then as well.

Now, $\operatorname{Mod}\left[S P_{1}\right]$ consists of all $\operatorname{Sig}\left[S P_{1}\right]$-algebras with an element on which $f$ yields $b$ and $g$ yields $c$. Consequently, given the axiom added in $S P, S P \models b=c$. However, since any $\operatorname{Sig}\left[S P_{1}\right]$-algebra is a subalgebra of an algebra in $\operatorname{Mod}\left[S P_{1}\right]$, the equational theory $T h\left(S P_{1}\right)$ is trivial (i.e., generated by the empty set). Hence $\mathcal{T}_{\text {INS }}\left(S P_{1}\right)$ consists of equational tautologies only as well, and $\mathcal{T}_{\text {INS }}(S P)=C l_{S i g[S P]}(\{\forall x: s \bullet f(x)=g(x)\})$ does not contain $b=c$.

However, under additional requirements on the underlying institution, completeness holds.

Theorem 4.4. Suppose that INS is $\langle\mathcal{H}, \mathcal{W}\rangle$-exact and admits parameterised $\langle\mathcal{H}, \mathcal{W}\rangle$ interpolation. Then $\mathcal{T}_{\text {INS }}$ is complete for specifications built from flat specifications using union, translation and hiding.

Proof. Assume that $S P \models \varphi$ for a specification $S P$ built from flat specifications using union, translation and hiding, with $\operatorname{Sig}[S P]=\Sigma$ and $\varphi \in \operatorname{Sen}(\Sigma)$. We show that $\varphi \in$ $\mathcal{T}_{\text {INS }}(S P)$ by induction on the structure of $S P$, and this shows completeness since $\mathcal{T}_{\text {INS }}$ is sound by Prop. 4.2:

— Let $S P$ be $\langle\Sigma, \Phi\rangle$ for $\Phi \subseteq \operatorname{Sen}(\Sigma)$. Then $\left.\Phi\right|_{\Sigma} \varphi$ and so $\varphi \in C l_{\Sigma}(\Phi)=\mathcal{T}_{\text {INS }}(\langle\Sigma, \Phi\rangle)$.

— Let $S P$ be $S P^{\prime}$ hide via $\sigma$ for some specification $S P^{\prime}$ with $\operatorname{Sig}\left[S P^{\prime}\right]=\Sigma^{\prime}$ and $\sigma: \Sigma \rightarrow$ $\Sigma^{\prime}$ in $\mathcal{H}$. Then $S P^{\prime} \models \sigma(\varphi)$. By the inductive hypothesis, $\sigma(\varphi) \in \mathcal{T}_{\text {INS }}\left(S P^{\prime}\right)$, and so $\varphi \in \sigma^{-1}\left(\mathcal{T}_{\text {INS }}\left(S P^{\prime}\right)\right)=\mathcal{T}_{\text {INS }}(S P)$.

— Let $S P$ be $S P^{\prime}$ with $\sigma$ for some specification $S P^{\prime}$ with $\operatorname{Sig}\left[S P^{\prime}\right]=\Sigma^{\prime}$ and $\sigma: \Sigma^{\prime} \rightarrow$ $\Sigma$ in $\mathcal{W}$. By Thm. 3.1, $S P^{\prime} \equiv\left\langle\Sigma_{1}, \Phi_{1}\right\rangle$ hide via $\sigma_{1}$ for some $\Sigma_{1} \in|\operatorname{Sign}|, \Phi_{1} \subseteq$ $\operatorname{Sen}\left(\Sigma_{1}\right)$, and $\sigma_{1}: \Sigma^{\prime} \rightarrow \Sigma_{1}$ in $\mathcal{H}$. Then, as in the (omitted, but well-known) proof of

\footnotetext{
${ }^{3}$ In particular, the missing signature morphism in the definition of $S P_{1}$ is the inclusion from $\operatorname{Sig}\left[S P_{0}\right] \backslash\{a: s\}$ to $\operatorname{Sig}\left[S P_{0}\right]$, and the definition of $S P$ abbreviates spec $S P=$ $S P_{1} \cup\left\langle\operatorname{Sig}\left[S P_{1}\right],\{\forall x: s \bullet f(x)=g(x)\}\right\rangle$.
} 
Thm. 3.1, $S P \equiv\left\langle\widehat{\Sigma}, \sigma^{\prime}\left(\Phi_{1}\right)\right\rangle$ hide via $\sigma_{1}^{\prime}$, where the following is a pushout in Sign:

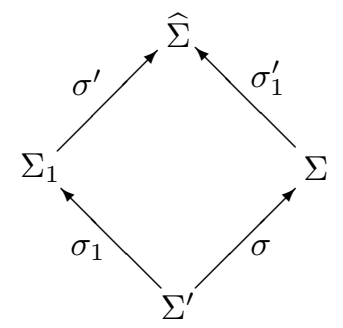

with $\sigma^{\prime} \in \mathcal{W}, \sigma_{1}^{\prime} \in \mathcal{H}$. Then $S P \models \varphi$ implies $\sigma^{\prime}\left(\Phi_{1}\right) \models_{\widehat{\Sigma}} \sigma_{1}^{\prime}(\varphi)$. Hence, by Craig $\langle\mathcal{H}, \mathcal{W}\rangle$-interpolation (the stronger, parameterised version is not needed in this case), there is an interpolant set $\Psi \subseteq \operatorname{Sen}\left(\Sigma^{\prime}\right)$ such that $\Phi_{1} \models_{\Sigma_{1}} \sigma_{1}(\Psi)$ and $\sigma(\Psi) \models_{\Sigma} \varphi$. The former yields $S P^{\prime}=\Psi$, so by the inductive hypothesis $\Psi \subseteq \mathcal{T}_{\text {INS }}\left(S P^{\prime}\right)$, and by the latter $\varphi \in C l_{\Sigma}(\sigma(\Psi)) \subseteq C l_{\Sigma}\left(\sigma\left(\mathcal{T}_{\text {INS }}\left(S P^{\prime}\right)\right)\right)=\mathcal{T}_{\text {INS }}(S P)$.

- Let $S P$ be $S P_{1} \cup S P_{2}$ for specifications $S P_{1}$ and $S P_{2}$ with $\operatorname{Sig}\left[S P_{1}\right]=\operatorname{Sig}\left[S P_{2}\right]=\Sigma$. By Thm. 3.1, $S P_{1} \equiv\left\langle\Sigma_{1}, \Phi_{1}\right\rangle$ hide via $\sigma_{1}$ for some $\Sigma_{1} \in|\operatorname{Sign}|, \Phi_{1} \subseteq \operatorname{Sen}\left(\Sigma_{1}\right)$ and $\sigma_{1}: \Sigma \rightarrow \Sigma_{1}$ in $\mathcal{H}$, and $S P_{2} \equiv\left\langle\Sigma_{2}, \Phi_{2}\right\rangle$ hide via $\sigma_{2}$ for some $\Sigma_{2} \in|\operatorname{Sign}|, \Phi_{2} \subseteq$ $\operatorname{Sen}\left(\Sigma_{2}\right)$ and $\sigma_{2}: \Sigma \rightarrow \Sigma_{2}$ in $\mathcal{H} \subseteq \mathcal{W}$. Then $S P \equiv\left\langle\widehat{\Sigma},\left\{\sigma_{2}^{\prime}\left(\Phi_{1}\right), \sigma_{1}^{\prime}\left(\Phi_{2}\right)\right\}\right\rangle$ hide via $\sigma_{2} ; \sigma_{1}^{\prime}$, as in the (omitted) proof of Thm. 3.1, where the following is a pushout in Sign:

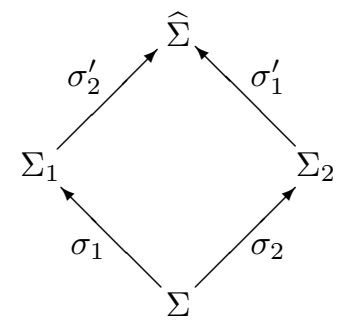

with $\sigma_{1}^{\prime} \in \mathcal{H}, \sigma_{2}^{\prime} \in \mathcal{W} . S P \models \varphi$ implies $\sigma_{2}^{\prime}\left(\Phi_{1}\right), \sigma_{1}^{\prime}\left(\Phi_{2}\right)=_{\widehat{\Sigma}} \sigma_{1}^{\prime}\left(\sigma_{2}(\varphi)\right)$. Then, by the parameterised $\langle\mathcal{H}, \mathcal{W}\rangle$-interpolation property, there is an interpolant set $\Psi \subseteq \operatorname{Sen}(\Sigma)$ such that $\Phi_{1} \models_{\Sigma_{1}} \sigma_{1}(\Psi)$ and $\Phi_{2} \cup \sigma_{2}(\Psi) \models_{\Sigma} \sigma_{2}(\varphi)$. The former yields $S P_{1} \models \Psi$, so by the inductive hypothesis, $\Psi \subseteq \mathcal{T}_{\text {INS }}\left(S P_{1}\right) \subseteq \mathcal{T}_{\text {INS }}(S P)$. By the latter, considering the pushout:

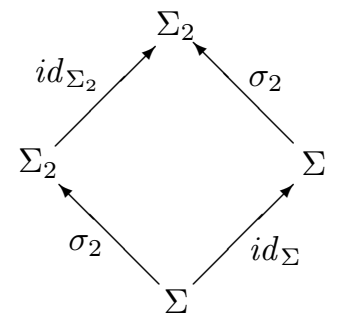

the parameterised $\langle\mathcal{H}, \mathcal{W}\rangle$-interpolation property yields an interpolant set $\Psi^{\prime} \subseteq \operatorname{Sen}(\Sigma)$ (for $\Phi_{2}$ and $\varphi$ w.r.t. $\Psi$ ) such that $\Phi_{2} \models \sigma_{2}\left(\Psi^{\prime}\right)$ and $\Psi^{\prime} \cup \Psi \models \varphi$. Since now the former yields $S P_{2} \models \Psi^{\prime}$, by the inductive hypothesis we have $\Psi^{\prime} \subseteq \mathcal{T}_{\text {INS }}\left(S P_{2}\right) \subseteq \mathcal{T}_{\text {INS }}(S P)$, and so we also get $\varphi \in C l_{\Sigma}\left(\mathcal{T}_{\text {INS }}(S P)\right)=\mathcal{T}_{\text {INS }}(S P)$. 
We mimic above the proof in (Borzyszkowski, 2002), which in turn largely followed (Bergstra et al., 1990) where the role of first-order interpolation for such results was perhaps first explored. However, we use a stronger (parameterised) interpolation property instead of Craig interpolation together with an assumption that the underlying institution is compact and has conjunction and implication. The latter idea was used for instance in (Diaconescu, 2008) to show completeness of a (stronger) calculus for proving entailment in a context of structured specifications, where the case of specifications built using union was simpler.

Another result of this kind, for a somewhat different collection of specification-building operations, is given in (Goguen and Roşu, 2004). They show soundness and completeness of $\mathcal{T}_{\text {INS }}$ with respect to a semantics which in essence calculates a normal form of specification expressions (see Thm. 3.1) but rely on conservativity of modules - see the footnote at the end of Sect. 2 - rather than on the strictly weaker requirement of parameterised interpolation. ${ }^{4}$

It is easy to see that the parameterised interpolation property is necessary for the above completeness result:

Proposition 4.5. Suppose that INS is an $\langle\mathcal{H}, \mathcal{W}\rangle$-exact institution such that $\mathcal{T}_{\text {INS }}$ is complete for specifications built from flat specifications using union, translation and hiding in INS. Then INS admits parameterised $\langle\mathcal{H}, \mathcal{W}\rangle$-interpolation.

Proof. Consider any pushout diagram in Sign

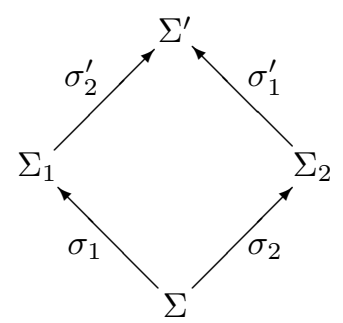

with $\sigma_{1}, \sigma_{1}^{\prime} \in \mathcal{H}, \sigma_{2}, \sigma_{2}^{\prime} \in \mathcal{W}$, and $\Phi_{1} \subseteq \operatorname{Sen}\left(\Sigma_{1}\right), \Phi_{2} \subseteq \operatorname{Sen}\left(\Sigma_{2}\right)$ and $\varphi_{2} \in \operatorname{Sen}\left(\Sigma_{2}\right)$ such that $\sigma_{2}^{\prime}\left(\Phi_{1}\right) \cup \sigma_{1}^{\prime}\left(\Phi_{2}\right) \models \sigma_{1}^{\prime}\left(\varphi_{2}\right)$. Let $S P \in \operatorname{Spec} c^{U T H}\left(\Sigma_{2}\right)$ be the specification $\left(\left(\left\langle\Sigma_{1}, \Phi_{1}\right\rangle\right.\right.$ hide via $\left.\sigma_{1}\right)$ with $\left.\sigma_{2}\right) \cup$ $\left\langle\Sigma_{2}, \Phi_{2}\right\rangle$. Then

$$
\begin{aligned}
\mathcal{T}_{\text {INS }}(S P) & =C l_{\Sigma_{2}}\left(C l_{\Sigma_{2}}\left(\sigma_{2}\left(\mathcal{T}_{\text {INS }}\left(\left\langle\Sigma_{1}, \Phi_{1}\right\rangle \text { hide via } \sigma_{1}\right)\right)\right) \cup \Phi_{2}\right) \\
& =C l_{\Sigma_{2}}\left(\sigma_{2}\left(\mathcal{T}_{\text {INS }}\left(\left\langle\Sigma_{1}, \Phi_{1}\right\rangle \text { hide via } \sigma_{1}\right)\right) \cup \Phi_{2}\right) .
\end{aligned}
$$

If $\mathcal{T}_{\text {INS }}$ is complete then $\varphi_{2} \in T h(S P)=\mathcal{T}_{\text {INS }}(S P)$, and we can take $\Phi=\mathcal{T}_{\text {INS }}\left(\left\langle\Sigma_{1}, \Phi_{1}\right\rangle\right.$ hide via $\left.\sigma_{1}\right)$ to be a set of interpolants for $\Phi_{1}$ and $\varphi_{2}$ w.r.t. $\Phi_{2}$.

\footnotetext{
${ }^{4}$ Contrary to a claim in the abstract of (Goguen and Roşu, 2004), conservativity is not a necessary condition for the results there. In fact, they give no technical statement that repeats this claim; they merely show that completeness fails in certain non-conservative examples.
} 


\section{Comparing property-oriented semantics}

As can be seen from Thm. 4.4 and Prop. 4.5, $\mathcal{T}_{\text {INS }}$ is complete only under rather strong assumptions concerning the underlying logical system. Even though these hold for FOPEQ, the institution of first order logic (with classes $\mathcal{W}$ and $\mathcal{H}$ chosen, say, to be all injective signature morphisms) this is a rather rare situation and $\mathcal{T}_{\text {INS }}$ is incomplete in many typical institutions of practical importance, including $\mathbf{E Q}$ and $\mathbf{E} \mathbf{Q}^{n e}$ (see Example 4.3). There have been attempts to preserve compositionality and nevertheless ensure completeness (Mossakowski et al., 2006). However, we show below that to improve on $\mathcal{T}_{\text {INS }}$, at least some aspects of compositionality must be sacrificed.

Theorem 5.1. Consider two property-oriented semantics $\mathcal{T}$ and $\mathcal{T}^{\prime}$ for specifications constructed using a set of specification-building operations, including all flat specifications. Let $\mathcal{T}$ be sound, monotone and closed-complete. Let $\mathcal{T}^{\prime}$ be theory-oriented, sound, compositional and extensive. Then $\mathcal{T}$ is at least as strong as $\mathcal{T}^{\prime}$ : for every $S P$, $\mathcal{T}^{\prime}(S P) \subseteq \mathcal{T}(S P)$.

Proof. By induction on the structure of $S P$. For flat specifications, $\mathcal{T}^{\prime}(\langle\Sigma, \Phi\rangle) \subseteq$ $C l_{\Sigma}(\Phi)=\mathcal{T}(\langle\Sigma, \Phi\rangle)$ by soundness of $\mathcal{T}^{\prime}$ and flat-completeness of $\mathcal{T}$ (which is the same as closed-completeness for flat specifications).

More generally: consider any well-formed specification $\operatorname{sbo}\left(S P_{1}, \ldots, S P_{n}\right)$ with $\Sigma_{i}=$ $\operatorname{Sig}\left[S P_{i}\right]$, where $i=1, \ldots, n$ here and below, and suppose $\mathcal{T}^{\prime}\left(S P_{i}\right) \subseteq \mathcal{T}\left(S P_{i}\right)$. Since $\mathcal{T}^{\prime}$ is theory-oriented and extensive, $\mathcal{T}^{\prime}\left(\left\langle\Sigma_{i}, \mathcal{T}^{\prime}\left(S P_{i}\right)\right\rangle\right)=\mathcal{T}^{\prime}\left(S P_{i}\right)$; we also have $\mathcal{T}\left(\left\langle\Sigma_{i}, \mathcal{T}^{\prime}\left(S P_{i}\right)\right\rangle\right)=$ $\mathcal{T}^{\prime}\left(S P_{i}\right)$. Then, using subsequently compositionality of $\mathcal{T}^{\prime}$, soundness of $\mathcal{T}^{\prime}$, closedcompleteness of $\mathcal{T}$ for sbo, and monotonicity of $\mathcal{T}$ (and the inductive assumption):

$$
\begin{aligned}
\mathcal{T}^{\prime}\left(\operatorname{sbo}\left(S P_{1}, \ldots, S P_{n}\right)\right) \\
\quad=\mathcal{T}^{\prime}\left(\operatorname{sbo}\left(\left\langle\Sigma_{1}, \mathcal{T}^{\prime}\left(S P_{1}\right)\right\rangle, \ldots,\left\langle\Sigma_{n}, \mathcal{T}^{\prime}\left(S P_{n}\right)\right\rangle\right)\right) \\
\quad \subseteq T\left(\operatorname{sbo}\left(\left\langle\Sigma_{1}, \mathcal{T}^{\prime}\left(S P_{1}\right)\right\rangle, \ldots,\left\langle\Sigma_{n}, \mathcal{T}^{\prime}\left(S P_{n}\right)\right\rangle\right)\right) \\
\quad=\mathcal{T}\left(\operatorname{sbo}\left(\left\langle\Sigma_{1}, \mathcal{T}^{\prime}\left(S P_{1}\right)\right\rangle, \ldots,\left\langle\Sigma_{n}, \mathcal{T}^{\prime}\left(S P_{n}\right)\right\rangle\right)\right) \\
\quad \subseteq \mathcal{T}\left(\operatorname{sbo}\left(S P_{1}, \ldots, S P_{n}\right)\right)
\end{aligned}
$$

This completes the inductive step and the proof of the theorem.

Corollary 5.2. $\mathcal{T}_{\text {INS }}$ is at least as strong as any sound, compositional and extensive theory-oriented semantics for structured specifications built from flat specifications using union, translation and hiding.

Proof. Directly from Prop. 4.2 and Thm. 5.1.

The requirement that the theory-oriented semantics considered be extensive is perhaps the most surprising one. Informally, if we forget about some axioms, we should not be able to soundly get more consequences. However, this requirement cannot be dropped in general, as the following counterexample shows.

Example 5.3. Consider an institution $\mathbf{I N S}_{0}$ with signatures $\Sigma$ and $\Sigma^{\prime}$, and a signature morphism $\sigma: \Sigma \rightarrow \Sigma^{\prime}$. Let $\operatorname{Sen}_{0}(\Sigma)=\{\varphi\}$, $\operatorname{Sen}_{0}\left(\Sigma^{\prime}\right)=\{\varphi, \psi\}$, with $\sigma$-translation preserving $\varphi$, and let $\left|\operatorname{Mod}_{0}(\Sigma)\right|=\left|\operatorname{Mod}_{0}\left(\Sigma^{\prime}\right)\right|=\left\{M_{0}, M_{1}, M_{2}\right\}$, with the identity $\sigma$-reduct. Suppose $M_{0} \vDash \models \varphi, M_{1} \models \varphi, M_{2} \models \varphi, M_{0} \vDash \psi \psi, M_{1} \not \models \psi, M_{2} \models \psi$ (over appropriate 
signatures) and that we have a $\Sigma$-specification $S P_{b a d}$ with $\operatorname{Mod}\left[S P_{b a d}\right]=\left\{M_{2}\right\}$. Then let $\mathcal{T}^{\prime}$ be such that $\mathcal{T}^{\prime}\left(S P_{b a d}\right)=\{\varphi\}$ and $\mathcal{T}^{\prime}\left(S P_{b a d}\right.$ with $\left.\sigma\right)=\{\varphi, \psi\}$, and $\mathcal{T}^{\prime}$ forgets the axiom $\varphi$ in all flat specifications. We can then ensure that for all $\Sigma$-specifications $S P$, if $\varphi \in \mathcal{T}^{\prime}(S P)$ then $M_{1} \notin \operatorname{Mod}[S P]$, since very informally, if $\varphi$ cannot be put into the theory of a specification as an axiom, the only way it can be there is as a consequence of $\psi$. Then $\mathcal{T}^{\prime}$ is sound and compositional, but for the $\Sigma^{\prime}$-specification $S P_{b a d}$ with $\sigma$, it is stronger than the expected sound, monotone and closed-complete theory-oriented semantics $\mathcal{T}_{\mathbf{I N S}_{0}}$ that yields $\mathcal{T}_{\mathbf{I N S}_{0}}\left(S P_{\text {bad }}\right)=\{\varphi\}$ and $\mathcal{T}_{\mathbf{I N S}_{0}}\left(S P_{\text {bad }}\right.$ with $\left.\sigma\right)=\{\varphi\}$.

To make this fully specific, suppose that there are no other signatures and non-identity signature morphisms, and no other sentences and models. Define:

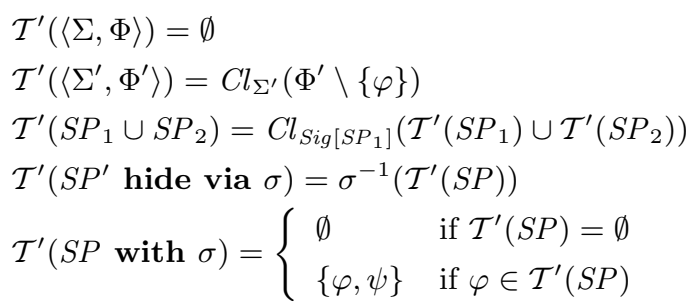

Put $S P_{b a d}=\left\langle\Sigma^{\prime},\{\psi\}\right\rangle$ hide via $\sigma$ and check that it has the properties required above. Indeed, $\mathcal{T}^{\prime}$ is a sound compositional theory-oriented semantics, but $\mathcal{T}^{\prime}\left(S P_{\text {bad }}\right.$ with $\left.\sigma\right)=$ $\{\varphi, \psi\}$ is a strictly larger theory than $\mathcal{T}_{\text {INS }_{0}}\left(S P_{\text {bad }}\right.$ with $\left.\sigma\right)=\{\varphi\}$.

The requirement that the semantics considered be theory-oriented cannot be dropped either:

Example 5.4. Building on Example 5.3, consider an institution $\mathbf{I N S}_{1}$ with exactly two signatures $\Sigma$ and $\Sigma^{\prime}$, and $\sigma: \Sigma \rightarrow \Sigma^{\prime}$ as the only non-identity signature morphism. Let $\operatorname{Sen}_{1}(\Sigma)=\left\{\varphi, \varphi^{\prime}\right\}, \operatorname{Sen}_{1}\left(\Sigma^{\prime}\right)=\left\{\varphi, \varphi^{\prime}, \psi\right\}$, with $\sigma$-translation preserving $\varphi$ and $\varphi^{\prime}$, and let $\left|\operatorname{Mod}_{1}(\Sigma)\right|=\left|\operatorname{Mod}_{1}\left(\Sigma^{\prime}\right)\right|=\left\{M_{0}, M_{1}, M_{2}, M_{3}\right\}$, with the identity $\sigma$-reduct. Define $M_{0} \not \models \varphi^{\prime}, M_{1} \models \varphi^{\prime}, M_{2} \models \varphi^{\prime}, M_{3} \models \varphi^{\prime}, M_{0} \not \models \varphi, M_{1} \not \models \varphi, M_{2} \models \varphi, M_{3} \models \varphi$, and $M_{0} \not \models \psi, M_{1} \not \models \psi, M_{2} \not \models \psi, M_{3} \models \psi$ (over appropriate signatures).

\begin{tabular}{|c|c|c|c|}
\hline$\models$ & $\varphi^{\prime}$ & $\varphi$ & $\psi$ \\
\hline$M_{0}$ & - & - & - \\
\hline$M_{1}$ & + & - & - \\
\hline$M_{2}$ & + & + & - \\
\hline$M_{3}$ & + & + & + \\
\hline
\end{tabular}

So, over the appropriate signatures we have $\varphi \models \varphi^{\prime}$, and $\psi \models \varphi$.

Now define a property-oriented semantics $\mathcal{T}^{\prime \prime}$ using inductive clauses that essentially copy those for $\mathcal{T}_{\mathbf{I N S}_{1}}$, except that for the flat $\Sigma^{\prime}$-specification with $\psi$ as the only axiom we omit exactly one of its consequences $\varphi^{\prime}$, and then for translation along $\sigma$ when the properties of the argument specification given by the semantics include $\varphi$ but not $\varphi^{\prime}$, we add $\psi$ as a property of the translated specification. The latter happens only if the specification to be translated along $\sigma$ results from hiding w.r.t. $\sigma$ of a specification with $\psi$ as the only axiom. 


$$
\begin{aligned}
& \mathcal{T}^{\prime \prime}(\langle\Sigma, \Phi\rangle)=C l_{\Sigma}(\Phi) \\
& \mathcal{T}^{\prime \prime}\left(\left\langle\Sigma^{\prime}, \Phi^{\prime}\right\rangle\right)= \begin{cases}\{\psi, \varphi\} & \text { if } \Phi^{\prime}=\{\psi\} \\
C l_{\Sigma^{\prime}}\left(\Phi^{\prime}\right) & \text { otherwise }\end{cases} \\
& \mathcal{T}^{\prime \prime}\left(S P_{1} \cup S P_{2}\right)=C l_{S i g\left[S P_{1}\right]}\left(\mathcal{T}^{\prime \prime}\left(S P_{1}\right) \cup \mathcal{T}^{\prime \prime}\left(S P_{2}\right)\right) \\
& \mathcal{T}^{\prime \prime}\left(S P^{\prime} \text { hide via } \sigma\right)=\sigma^{-1}\left(\mathcal{T}^{\prime \prime}\left(S P^{\prime}\right)\right) \\
& \mathcal{T}^{\prime \prime}(S P \text { with } \sigma)= \begin{cases}C l_{\Sigma^{\prime}}\left(\sigma\left(\mathcal{T}^{\prime \prime}(S P)\right)\right) & \text { if } \varphi^{\prime} \in \mathcal{T}^{\prime \prime}(S P) \text { or } \varphi \notin \mathcal{T}^{\prime \prime}(S P) \\
C l_{\Sigma^{\prime}}\left(\sigma\left(\mathcal{T}^{\prime \prime}(S P)\right) \cup\{\psi\}\right) & \text { if } \varphi^{\prime} \notin \mathcal{T}^{\prime \prime}(S P) \text { and } \varphi \in \mathcal{T}^{\prime \prime}(S P)\end{cases}
\end{aligned}
$$

$\mathcal{T}^{\prime \prime}$ is a sound, compositional, extensive property-oriented semantics. It is not theoryoriented though, since in particular $\mathcal{T}^{\prime \prime}\left(\left\langle\Sigma^{\prime},\{\psi\}\right\rangle\right)=\{\psi, \varphi\}$ is not closed under consequence (it does not contain $\varphi^{\prime}$ ). Since for all other $\Sigma^{\prime}$-specifications and for flat $\Sigma$ specifications the semantics $\mathcal{T}^{\prime \prime}$ yields a theory, this is exploited to "enlarge our knowledge" about $\Sigma$-specifications $S P$ with $\mathcal{T}^{\prime \prime}(S P)$ containing $\varphi$ but not $\varphi^{\prime}$. Namely, as in Example 5.3, putting $S P_{b a d}=\left\langle\Sigma^{\prime},\{\psi\}\right\rangle$ hide via $\sigma$, we get $\mathcal{T}^{\prime \prime}\left(S P_{b a d}\right)=\{\varphi\}$ (while $\left.\mathcal{T}_{\text {INS }_{1}}\left(S P_{b a d}\right)=\left\{\varphi, \varphi^{\prime}\right\}\right)$, and so $\mathcal{T}^{\prime \prime}\left(S P_{b a d}\right.$ with $\left.\sigma\right)=\left\{\psi, \varphi, \varphi^{\prime}\right\}$, which is a strictly larger theory than $\mathcal{T}_{\text {INS }_{1}}\left(S P_{\text {bad }}\right.$ with $\left.\sigma\right)=\left\{\varphi, \varphi^{\prime}\right\}$.

The counterexample property-oriented semantics given in Example 5.4 is compositional but not monotone. This is necessarily so, since for the semantics considered in Thm. 5.1 and Cor. 5.2, if we assume that it is monotone then the requirement that it be theoryoriented may be dropped:

Theorem 5.5. Consider two property-oriented semantics $\mathcal{T}$ and $\mathcal{T}^{\prime}$ for specifications constructed using a set of specification-building operations, including all flat specifications. Let $\mathcal{T}$ be sound, monotone and closed-complete. Let $\mathcal{T}^{\prime}$ be sound, monotone and extensive. Then $\mathcal{T}$ is at least as strong as $\mathcal{T}^{\prime}$ : for every $S P, \mathcal{T}^{\prime}(S P) \subseteq \mathcal{T}(S P)$.

Proof. By induction on the structure of $S P$. For flat specifications, $\mathcal{T}^{\prime}(\langle\Sigma, \Phi\rangle) \subseteq$ $C l_{\Sigma}(\Phi)=\mathcal{T}(\langle\Sigma, \Phi\rangle)$ by soundness of $\mathcal{T}^{\prime}$ and flat-completeness of $\mathcal{T}$ (which is the same as closed-completeness for flat specifications).

More generally: consider any well-formed specification $\operatorname{sbo}\left(S P_{1}, \ldots, S P_{n}\right)$ with $\Sigma_{i}=$ $\operatorname{Sig}\left[S P_{i}\right]$, where $i=1, \ldots, n$ here and below, and suppose $\mathcal{T}^{\prime}\left(S P_{i}\right) \subseteq \mathcal{T}\left(S P_{i}\right)$ (inductive assumption). Since $\mathcal{T}^{\prime}$ is extensive, we have $\mathcal{T}^{\prime}\left(S P_{i}\right) \subseteq \mathcal{T}^{\prime}\left(\left\langle\Sigma_{i}, \mathcal{T}^{\prime}\left(S P_{i}\right)\right\rangle\right)$. Also: $\mathcal{T}\left(\left\langle\Sigma_{i}, \mathcal{T}^{\prime}\left(S P_{i}\right)\right\rangle\right)=C l_{\Sigma_{i}}\left(\mathcal{T}^{\prime}\left(S P_{i}\right)\right) \subseteq C l_{\Sigma_{i}}\left(\mathcal{T}\left(S P_{i}\right)\right)=\mathcal{T}\left(S P_{i}\right)$ by flat-completeness of $\mathcal{T}$, the inductive assumption, and closure of $\mathcal{T}(S P)$ under consequence $(\mathcal{T}$ is closed-complete, hence theory-oriented). Then, using subsequently monotonicity of $\mathcal{T}^{\prime}$, soundness of $\mathcal{T}^{\prime}$, closed-completeness of $\mathcal{T}$ for sbo, and monotonicity of $\mathcal{T}$ :

$$
\begin{aligned}
\mathcal{T}^{\prime}\left(\operatorname{sbo}\left(S P_{1}, \ldots, S P_{n}\right)\right) \\
\quad \subseteq \mathcal{T}^{\prime}\left(\operatorname{sbo}\left(\left\langle\Sigma_{1}, \mathcal{T}^{\prime}\left(S P_{1}\right)\right\rangle, \ldots,\left\langle\Sigma_{n}, \mathcal{T}^{\prime}\left(S P_{n}\right)\right\rangle\right)\right) \\
\quad \subseteq T h\left(\operatorname{sbo}\left(\left\langle\Sigma_{1}, \mathcal{T}^{\prime}\left(S P_{1}\right)\right\rangle, \ldots,\left\langle\Sigma_{n}, \mathcal{T}^{\prime}\left(S P_{n}\right)\right\rangle\right)\right) \\
\quad=\mathcal{T}\left(\operatorname{sbo}\left(\left\langle\Sigma_{1}, \mathcal{T}^{\prime}\left(S P_{1}\right)\right\rangle, \ldots,\left\langle\Sigma_{n}, \mathcal{T}^{\prime}\left(S P_{n}\right)\right\rangle\right)\right) \\
\quad \subseteq \mathcal{T}\left(\operatorname{sbo}\left(S P_{1}, \ldots, S P_{n}\right)\right)
\end{aligned}
$$

This completes the inductive step and the proof of the theorem. 
Corollary 5.6. $\mathcal{T}_{\text {INS }}$ is at least as strong as any sound, monotone and extensive propertyoriented semantics for structured specifications built from flat specifications using union, translation and hiding.

Proof. Directly from Prop. 4.2 and Thm. 5.5.

Example 5.3 shows that the requirement that the semantics considered in the above corollary be extensive cannot be dropped: the counterexample semantics $\mathcal{T}^{\prime}$ given there is monotone.

The above analysis of the relative power of property-oriented semantics for structured specifications was based on an implicit assumption that there are no signatures, sentences, and specifications other than those we are dealing with. In a way, this is a version of the famous closed world assumption. In particular, Examples 5.3 and 5.4 relied on this to justify soundness of the counterexample semantics constructed there that for some specifications (built from flat specifications using union, translation and hiding) yield a theory that is properly richer than the theory produced by the standard compositional semantics. Consequently, the counterexamples do not apply if we consider the semantics for specifications in some potential extensions of the specification framework.

As before, consider a class Spec of specifications built using a family of specificationbuilding operations. For any class $\mathcal{S P}$ of new specification constants, with model-theoretic semantics given as usual (i.e., for each $S P \in \mathcal{S P}$ we have $\operatorname{Sig}[S P] \in \mid$ Sign $\mid$ and $\operatorname{Mod}[S P] \subseteq$ $|\operatorname{Mod}(\operatorname{Sig}[S P])|$, hence we also have $T h(S P)=\operatorname{Th}(\operatorname{Mod}[S P]))$, let $\operatorname{Spec}(\mathcal{S P})$ be the class of specifications that contains Spec and $\mathcal{S P}$ and is closed under the specification-building operations considered, with a semantics that extends the semantics for specifications in Spec and $\mathcal{S P}$ using the meaning of the specification-building operations as explained in Sect. 3.

We say that a property-oriented semantics $\mathcal{T}$ for specifications in Spec is persistently sound and compositional if for any class of new specification constants $\mathcal{S} \mathcal{P}_{\text {new }}$ with modelclass semantics, for any sound property-oriented meaning for specifications in $\mathcal{S P}_{\text {new }}$ given by $\mathcal{T}_{\text {new }}(S P) \subseteq T h(S P)$ for all $S P \in \mathcal{S P}_{\text {new }}$, there is a sound and compositional property-oriented semantics $\widehat{\mathcal{T}}$ for $\operatorname{Spec}\left(\mathcal{S P} \mathcal{P}_{\text {new }}\right)$ that extends $\mathcal{T}$ and $\mathcal{T}_{\text {new }}$, that is such that $\widehat{\mathcal{T}}(S P)=\mathcal{T}(S P)$ for $S P \in S p e c$ and $\widehat{\mathcal{T}}(S P)=\mathcal{T}_{\text {new }}(S P)$ for $S P \in \mathcal{S P} \mathcal{P}_{\text {new }}$. Clearly, any persistently sound and compositional property-oriented semantics is sound and compositional, but the opposite implication fails in general.

It is easy to check that the standard compositional property-oriented semantics $\mathcal{T}_{\text {INS }}$ for specifications built from flat specifications using union, translation and hiding is persistently sound and compositional. Moreover, it is the strongest such property-oriented semantics. In contrast to the previous results, this does not require the semantics considered to be extensive.

Theorem 5.7. Consider two property-oriented semantics $\mathcal{T}$ and $\mathcal{T}^{\prime}$ for specifications constructed using a set of specification-building operations, including all flat specifications. Let $\mathcal{T}$ be sound, monotone and closed-complete. Let $\mathcal{T}^{\prime}$ be persistently sound and compositional. Then $\mathcal{T}$ is at least as strong as $\mathcal{T}^{\prime}$ : for every $S P, \mathcal{T}^{\prime}(S P) \subseteq \mathcal{T}(S P)$. 
Proof. We proceed by induction on the structure of specifications. Consider a specification $\operatorname{sbo}\left(S P_{1}, \ldots, S P_{n}\right), n \geq 0$, where $\mathcal{T}^{\prime}\left(S P_{1}\right) \subseteq \mathcal{T}\left(S P_{1}\right), \ldots, \mathcal{T}^{\prime}\left(S P_{n}\right) \subseteq \mathcal{T}\left(S P_{n}\right)$. We need to show that $\mathcal{T}^{\prime}\left(\mathbf{s b o}\left(S P_{1}, \ldots, S P_{n}\right)\right) \subseteq \mathcal{T}\left(\mathbf{s b o}\left(S P_{1}, \ldots, S P_{n}\right)\right)$.

Let $S P_{1}^{\prime}, \ldots, S P_{n}^{\prime}$ be new specification constants with semantics given by $\operatorname{Sig}\left[S P_{1}^{\prime}\right]=$ $\operatorname{Sig}\left[S P_{1}\right], \operatorname{Mod}\left[S P_{1}^{\prime}\right]=\operatorname{Mod}\left[\left\langle\operatorname{Sig}\left[S P_{1}\right], \mathcal{T}^{\prime}\left(S P_{1}\right)\right\rangle\right], \ldots, \operatorname{Sig}\left[S P_{n}^{\prime}\right]=\operatorname{Sig}\left[S P_{n}\right], \operatorname{Mod}\left[S P_{n}^{\prime}\right]=$ $\operatorname{Mod}\left[\left\langle\operatorname{Sig}\left[S P_{n}\right], \mathcal{T}^{\prime}\left(S P_{n}\right)\right\rangle\right]$, and property-oriented meaning $\mathcal{T}_{\text {new }}\left(S P_{1}^{\prime}\right)=\mathcal{T}^{\prime}\left(S P_{1}\right), \ldots$, $\mathcal{T}_{\text {new }}\left(S P_{n}^{\prime}\right)=\mathcal{T}^{\prime}\left(S P_{n}\right)$.

Since $\mathcal{T}^{\prime}$ is persistently sound and compositional, there is a sound and compositional property-oriented semantics $\widehat{\mathcal{T}^{\prime}}$ that extends $\mathcal{T}^{\prime}$ and $\mathcal{T}_{\text {new }}$ to $\operatorname{Spec}\left(\left\{S P_{1}^{\prime}, \ldots, S P_{n}^{\prime}\right\}\right)$. Then, using compositionality of $\widehat{\mathcal{T}^{\prime}}$, its soundness, the definition of the model-class semantics of the new constants, closed-completeness of $\mathcal{T}$, and finally extensiveness (which follows from flat-completeness, implied by closed-completeness) and monotonicity of $\mathcal{T}$ :

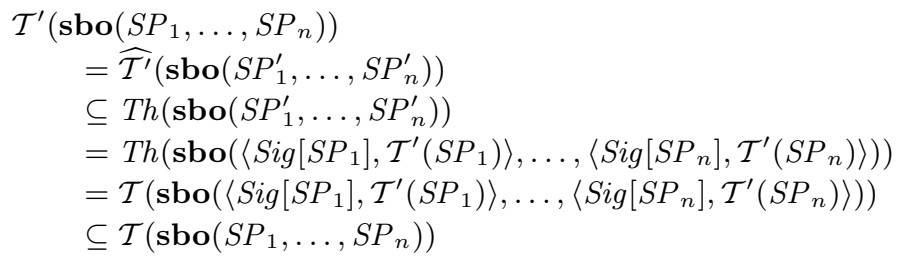

Corollary 5.8. $\mathcal{T}_{\text {INS }}$ is at least as strong as any persistently sound and compositional property-oriented semantics for structured specifications built from flat specifications using union, translation and hiding.

Proof. Directly from Prop. 4.2 and Thm. 5.7.

\section{Entailment systems}

The notion of an institution as recalled in Sect. 2 captures model-theoretic aspects of logical systems. An institution is typically augmented by an entailment system that approximates the semantic consequence relation, and in this section we consider the consequences of the results above in this setting. Entailment systems are normally defined with reference to a set of proof rules, but the presentation here abstracts away from this level of detail.

An entailment relation on a set $\mathbb{S}$ of sentences is a binary relation $\vdash \subseteq \mathcal{P}(\mathbb{S}) \times \mathbb{S}$ satisfying the following properties:

reflexivity: $\{\varphi\} \vdash \varphi$;

weakening: if $\Phi \vdash \varphi$ then $\Phi \cup \Psi \vdash \varphi$; and

transitivity: if $\Phi \vdash \psi$ and $\Psi_{\varphi} \vdash \varphi$ for each $\varphi \in \Phi$ then $\bigcup_{\varphi \in \Phi} \Psi_{\varphi} \vdash \psi$

for all sentences $\varphi, \psi \in \mathbb{S}$ and sets of sentences $\Phi, \Psi \subseteq \mathbb{S}$ and $\Psi_{\varphi} \subseteq \mathbb{S}$ for $\varphi \in \Phi$.

Clearly, the semantic consequence relation defined in Sect. 2 is a entailment relation in the above sense.

Let Sen: Sign $\rightarrow$ Set be a functor. An entailment system for Sen is a family of 
entailment relations $\mathcal{E}=\left\langle\vdash_{\Sigma} \subseteq \mathcal{P}(\operatorname{Sen}(\Sigma)) \times \operatorname{Sen}(\Sigma)\right\rangle_{\Sigma \in|\operatorname{Sign}|}$ such that for each morphism $\sigma: \Sigma \rightarrow \Sigma^{\prime}$ in $\operatorname{Sign}$, sentence $\varphi \in \operatorname{Sen}(\Sigma)$ and set $\Phi \subseteq \operatorname{Sen}(\Sigma)$, if $\Phi \vdash_{\Sigma} \varphi$ then $\operatorname{Sen}(\sigma)(\Phi) \vdash_{\Sigma^{\prime}} \operatorname{Sen}(\sigma)(\varphi)$, where $\operatorname{Sen}(\sigma)(\Phi)$ denotes the image of $\Phi$ under $\operatorname{Sen}(\sigma)$.

Given an institution INS $=\left\langle\right.$ Sign, Sen, $\left.\mathbf{M o d},\left\langle\models_{\Sigma}\right\rangle_{\Sigma \in \mid \text { Sign } \mid}\right\rangle$, an entailment system for INS (Meseguer, 1989; Harper et al., 1994) is an entailment system $\mathcal{E}=\left\langle\vdash_{\Sigma}\right\rangle_{\Sigma \in \mid \text { Sign }}$ for Sen that is sound with respect to semantic consequence, that is, for each signature $\Sigma$, $\Sigma$-sentence $\varphi \in \operatorname{Sen}(\Sigma)$ and set $\Phi \subseteq \operatorname{Sen}(\Sigma)$, if $\Phi \vdash_{\Sigma} \varphi$ then $\Phi \models_{\Sigma} \varphi$. Such an entailment system $\mathcal{E}$ is complete for INS if the opposite implication holds. Clearly, for any institution INS, the semantic consequence relations form an entailment system $\mathcal{E}_{\text {INS }}=$ $\left\langle=_{\Sigma}\right\rangle_{\Sigma \in \mid \text { Sign } \mid}$, which is sound and complete for INS.

A general logic (Meseguer, 1989) is an institution INS equipped with an entailment system $\mathcal{E}$ for INS.

For the rest of this section, let $\mathcal{E}=\left\langle\vdash_{\Sigma}\right\rangle_{\Sigma \in \mid \text { Sign } \mid}$ be an arbitrary entailment system for Sen : Sign $\rightarrow$ Set.

For any signature $\Sigma \in|\mathbf{S i g n}|$, a set of sentences $\Phi \subseteq \operatorname{Sen}(\Sigma)$ is an $\mathcal{E}$-theory if it is closed under $\vdash_{\Sigma}$ : if $\Phi \vdash_{\Sigma} \varphi$ then $\varphi \in \Phi$ for all $\varphi \in \operatorname{Sen}(\Sigma)$. For any set $\Phi \subseteq \operatorname{Sen}(\Sigma)$, the least $\mathcal{E}$-theory that contains $\Phi$ will be denoted by $C l_{\Sigma}^{\mathcal{E}}(\Phi)$. Clearly, for any institution INS and its semantic entailment system $\mathcal{E}_{\text {INS }}, C l_{\Sigma}^{\mathcal{E}_{\text {INS }}}\left({ }_{-}\right)$coincides with $C l_{\Sigma}\left({ }_{-}\right)$, and $\mathcal{E}_{\text {INS }}$-theories are exactly the theories in INS as defined in Sect. 2.

An entailment system $\mathcal{E}=\left\langle\vdash_{\Sigma}\right\rangle_{\Sigma \in \mid \text { Sign } \mid}$ for Sen: Sign $\rightarrow$ Set is trivial if for each signature $\Sigma \in|\operatorname{Sign}|, \vdash_{\Sigma}=\mathcal{P}(\operatorname{Sen}(\Sigma)) \times \operatorname{Sen}(\Sigma)$ (each set entails all sentences).

Proposition 6.1. Given an entailment system $\mathcal{E}$, if $\mathcal{E}$ is non-trivial then there is an

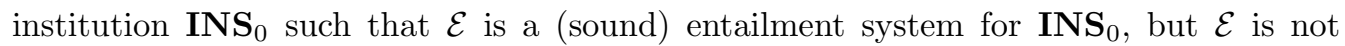
complete for $\mathbf{I N S}_{0}$. If $\mathcal{E}$ is trivial then it is complete for any institution for which it is sound.

Proof. A non-trivial entailment system is incomplete for an institution $\mathbf{I N S}_{0}$ in which

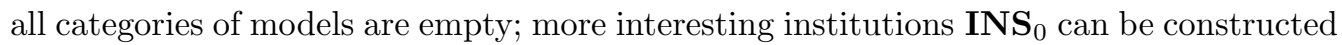
as well. The other part is trivial.

Proposition 6.2. For any entailment system $\mathcal{E}$ there is an institution $\mathbf{I N S}_{\mathcal{E}}$ such that $\mathcal{E}$ is (sound and) complete for $\mathbf{I N S}_{\mathcal{E}}$.

Proof. Consider an entailment system $\mathcal{E}=\left\langle\vdash_{\Sigma}\right\rangle_{\Sigma \in \mid \text { Sign } \mid}$ for Sen: Sign $\rightarrow$ Set. For any signature $\Sigma \in|\mathbf{S i g n}|$, define $\Sigma$-models to be $\mathcal{E}$-theories and satisfaction to be membership: $\operatorname{Mod}(\Sigma)=\left\{\Phi \subseteq \operatorname{Sen}(\Sigma) \mid C l_{\Sigma}^{\mathcal{E}}(\Phi)=\Phi\right\}$ (considered as a discrete category), and then for $M \in \operatorname{Mod}(\Sigma)$ and $\varphi \in \operatorname{Sen}(\Sigma)$ define $M \models_{\Sigma} \varphi$ to hold iff $\varphi \in M$. Furthermore, for any signature morphism $\sigma: \Sigma \rightarrow \Sigma^{\prime}$ define the reduct to be the coimage w.r.t. translation of sentences: for $M^{\prime} \in \operatorname{Mod}\left(\Sigma^{\prime}\right),\left.M^{\prime}\right|_{\sigma}=\sigma^{-1}\left(M^{\prime}\right)$. By preservation of entailment in $\mathcal{E}$ along signature morphisms, it follows that indeed $\left.M^{\prime}\right|_{\sigma} \in \operatorname{Mod}(\Sigma)$, and the satisfaction condition holds trivially. This defines an institution $\mathbf{I N S}_{\mathcal{E}}=\left\langle\operatorname{Sign}, \mathbf{S e n}, \mathbf{M o d},\left\langle\mid=_{\Sigma}\right\rangle_{\Sigma \in|\operatorname{Sign}|}\right\rangle$.

Now, given any set of $\Sigma$-sentences $\Phi \subseteq \operatorname{Sen}(\Sigma)$ and $\Sigma$-sentence $\varphi \in \operatorname{Sen}(\Sigma), \Phi \models \varphi$ in $\mathbf{I N S}_{\mathcal{E}}$ means that for all $M \in \operatorname{Mod}(\Sigma)$, if $\Phi \subseteq M$ then $\varphi \in M$, which is equivalent to $\varphi \in M_{\Phi}$, where $M_{\Phi}=C l_{\Sigma}^{\mathcal{E}}(\Phi)$ is the least model in $\operatorname{Mod}(\Sigma)$ that contains $\Phi$. Hence, $\Phi \models \varphi$ in $\mathbf{I N S}_{\mathcal{E}}$ iff $\Phi \vdash_{\Sigma} \varphi$. 
As remarked in Sect. 3, the syntax of flat specifications and union, translation and hiding introduced there for an arbitrary institution INS depends only on the category of signatures with distinguished class $\mathcal{H}$ and $\mathcal{W}$ of signature morphisms, satisfying the requirements imposed in Sect. 3, and the sentence functor Sen: Sign $\rightarrow$ Set. Consequently, we can consider such specifications whenever just an entailment system $\mathcal{E}$ for Sen together with appropriate $\mathcal{H}, \mathcal{W}$ is given, rather than an entire institution. In particular, the signature $\operatorname{Sig}[S P]$ of any specification $S P \in S p e c{ }^{U T H}$ is then well defined.

The concept of a property-oriented semantics directly carries over to this framework: as in Sect. 4, a property-oriented semantics is a function $\mathcal{T}$ that maps any specification $S P$ to a set of $\Sigma$-sentences $\mathcal{T}(S P) \subseteq \operatorname{Sen}(\operatorname{Sig}[S P])$.

Given such a property-oriented semantics, definitions of its monotonicity, compositionality and extensiveness carry over in a similarly straightforward way. We say that $\mathcal{T}$ is $\mathcal{E}$-theory-oriented if $\mathcal{T}(S P)$ is an $\mathcal{E}$-theory for all specifications $S P$.

However, concepts related to the model-theoretic part of the institution require more care.

A property-oriented semantics $\mathcal{T}$ is $\mathcal{E}$-sound if it is sound in any institution INS (with the same signature category and sentence functor as for $\mathcal{E}$ ) for which $\mathcal{E}$ is sound (or equivalently, in any general logic with $\mathcal{E}$ as the entailment system).

A sound property-oriented semantics $\mathcal{T}$ is $\mathcal{E}$-complete in a class of institutions $\mathcal{J N} \mathcal{N}$, if it is complete in any institution INS $\in \mathcal{J N} \mathcal{S}$ (with the same signature category and sentence functor as for $\mathcal{E}$ ) for which $\mathcal{E}$ is sound and complete. $\mathcal{E}$-closed-completeness and $\mathcal{E}$-flat-completeness may be defined analogously — we will not use these concepts here though.

$\mathcal{E}$-completeness is perhaps a weaker notion than one would expect: we might have required completeness of the semantics in any institution INS $\in \mathcal{J N} \mathcal{S}$ for which $\mathcal{E}$ is sound but not necessarily complete, so in any general logic with $\mathcal{E}$ as the entailment system. As can be derived from Prop. 6.1, such a stronger property would not be achievable at all though, unless the entailment system is trivial (or a very narrow class $\mathcal{J} \mathcal{N} S$ is considered).

The definition of the standard compositional theory-oriented semantics for specifications in $S p e c^{U T H}$ requires only an obvious tiny adjustment:

$$
\begin{aligned}
& \mathcal{T}_{\mathcal{E}}(\langle\Sigma, \Phi\rangle)=C l_{\Sigma}^{\mathcal{E}}(\Phi) \\
& \mathcal{T}_{\mathcal{E}}\left(S P \cup S P^{\prime}\right)=C l_{\text {Sig }[S P]}^{\mathcal{E}}\left(\mathcal{T}_{\mathcal{E}}(S P) \cup \mathcal{T}_{\mathcal{E}}\left(S P^{\prime}\right)\right) \\
& \mathcal{T}_{\mathcal{E}}(S P \text { with } \sigma: S i g[S P] \rightarrow \Sigma)=C l_{\Sigma}^{\mathcal{E}}\left(\sigma\left(\mathcal{T}_{\mathcal{E}}(S P)\right)\right) \\
& \mathcal{T}_{\mathcal{E}}(S P \text { hide via } \sigma: \Sigma \rightarrow \operatorname{Sig}[S P])=\sigma^{-1}\left(\mathcal{T}_{\mathcal{E}}(S P)\right)
\end{aligned}
$$

Proposition 6.3. $\mathcal{T}_{\mathcal{E}}$ is an $\mathcal{E}$-sound $\mathcal{E}$-theory-oriented semantics for specifications built from flat specifications using union, translation and hiding. It is monotone, compositional and extensive.

As for $\mathcal{T}_{\text {INS }}$ in Sect. 4, completeness does not hold, unless the class of institutions (general logics) considered is subject to further requirements:

Corollary 6.4. Let $\mathcal{J N} \mathcal{S}$ be the class of institutions that are $\langle\mathcal{H}, \mathcal{W}\rangle$-exact and admit parameterised $\langle\mathcal{H}, \mathcal{W}\rangle$-interpolation. Then $\mathcal{T}_{\mathcal{E}}$ is $\mathcal{E}$-complete for specifications built from flat specifications using union, translation and hiding in the class $\mathcal{I N S}$. 
Proof. Follows from Thm. 4.4.

Interpolation properties may be directly defined for an entailment system $\mathcal{E}=\left\langle\vdash_{\Sigma}\right\rangle_{\Sigma \in|\operatorname{Sign}|}$ for a sentence functor Sen: Sign $\rightarrow$ Set, without reference to the underlying institution. Namely, consider again the following commuting diagram in Sign:

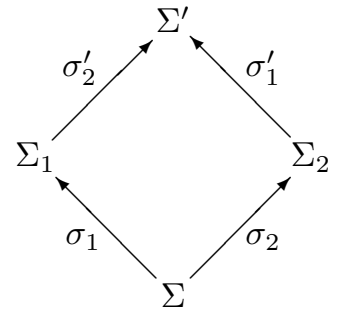

This diagram admits parameterised (or Craig-Robinson) interpolation if for any $\Phi_{1} \subseteq$ $\operatorname{Sen}\left(\Sigma_{1}\right), \Phi_{2} \subseteq \operatorname{Sen}\left(\Sigma_{2}\right)$ and $\varphi \in \operatorname{Sen}\left(\Sigma_{2}\right)$, whenever $\sigma_{2}^{\prime}\left(\Phi_{1}\right) \cup \sigma_{1}^{\prime}\left(\Phi_{2}\right) \vdash_{\Sigma^{\prime}} \sigma_{1}^{\prime}(\varphi)$ then for some $\Phi \subseteq \operatorname{Sen}(\Sigma)$ such that $\Phi_{1} \vdash_{\Sigma_{1}} \sigma_{1}(\Phi)$ we have $\Phi_{2} \cup \sigma_{2}(\Phi) \vdash_{\Sigma_{2}} \varphi$. The diagram admits Craig interpolation if it admits parameterised interpolation with "parameter set" $\Phi_{2}=\emptyset$.

Given classes $\mathcal{H}, \mathcal{W} \subseteq$ Sign of signature morphisms, we say that $\mathcal{E}$ admits parameterised (resp. Craig) $\langle\mathcal{H}, \mathcal{W}\rangle$-interpolation if for any signature morphisms $\delta \in \mathcal{H}$ and $\tau \in \mathcal{W}$ with a common source there are $\delta^{\prime} \in \mathcal{H}$ and $\tau^{\prime} \in \mathcal{W}$ forming a pushout in Sign

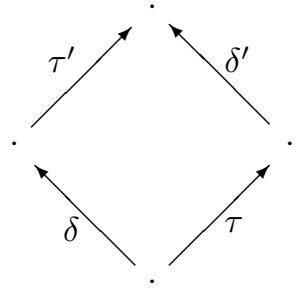

that admits parameterised (resp. Craig) interpolation; then any such pushout admits parameterised (resp. Craig) interpolation as well.

Clearly, if $\mathcal{E}$ is (sound and) complete for an institution INS then the above interpolation properties for $\mathcal{E}$ coincide with those for INS as defined in Sect. 2.

So, for an entailment system that admits parameterised $\langle\mathcal{H}, \mathcal{W}\rangle$-interpolation, the semantics $\mathcal{T}_{\mathcal{E}}$ is $\mathcal{E}$-complete for the class of institutions that are $\langle\mathcal{H}, \mathcal{W}\rangle$-exact.

Even though $\mathcal{T}_{\mathcal{E}}$ is not $\mathcal{E}$-complete in general, it is in essence the strongest compositional $\mathcal{E}$-theory oriented semantics:

Corollary 6.5. $\mathcal{T}_{\mathcal{E}}$ is at least as strong as any $\mathcal{E}$-sound, compositional, extensive $\mathcal{E}$ theory-oriented semantics for structured specifications built from flat specifications using union, translation and hiding.

Proof. Let $\mathcal{T}$ be an $\mathcal{E}$-sound, compositional, extensive $\mathcal{E}$-theory-oriented semantics. Consider the institution $\mathbf{I N S}_{\mathcal{E}}$ where semantic entailment coincides with entailment in $\mathcal{E}$, as given by Prop. 6.2. Then $\mathcal{T}_{\mathcal{E}}$ coincides with $\mathcal{T}_{\mathbf{I N S}_{\mathcal{E}}}$, and $\mathcal{T}$ is sound in $\mathbf{I N S}_{\mathcal{E}}$ (as well as compositional and extensive). Consequently, $\mathcal{T}_{\mathcal{E}}$ is at least as strong as $\mathcal{T}$ by Cor. 5.2. 
The assumption that the semantics considered be extensive cannot be dropped. Example 5.3 can be adapted to the framework of an entailment system:

Example 6.6. Consider an entailment system $\mathcal{E}^{0}$ that consists of semantic consequence for the institution constructed in Example 5.3. That is, take the category of signatures Sign $_{0}$ with exactly two objects $\Sigma$ and $\Sigma^{\prime}$, and $\sigma: \Sigma \rightarrow \Sigma^{\prime}$ as the only non-identity morphism. The sentence functor is given by $\operatorname{Sen}_{0}(\Sigma)=\{\varphi\}, \operatorname{Sen}_{0}\left(\Sigma^{\prime}\right)=\{\varphi, \psi\}$, with $\sigma$-translation preserving $\varphi$. Define $\mathcal{E}^{0}$ as the least entailment system for $\mathbf{S e n}_{0}$ such that $\psi \vdash_{\Sigma^{\prime}}^{0} \varphi$.

Then consider the property-oriented semantics $\mathcal{T}^{\prime}$ defined in Example 5.3. It is $\mathcal{E}^{0}$ sound (by the same reasoning as in Example 5.3), compositional and $\mathcal{E}^{0}$-theory-oriented. Moreover, for $S P_{b a d}$ defined as $\left\langle\Sigma^{\prime},\{\psi\}\right\rangle$ hide via $\sigma$, we have $\mathcal{T}^{\prime}\left(S P_{b a d}\right.$ with $\left.\sigma\right)=$ $\{\varphi, \psi\}$, while $\mathcal{T}_{\mathcal{E}^{0}}\left(S P_{\text {bad }}\right.$ with $\left.\sigma\right)=\{\varphi\}$.

Similarly, the assumption that the semantics considered be $\mathcal{E}$-theory-oriented cannot be dropped, since Example 5.4 can be adapted here as well:

Example 6.7. Consider an entailment system $\mathcal{E}^{1}$ that consists of semantic consequence for the institution constructed in Example 5.4. That is, take the category of signatures $\operatorname{Sign}_{1}$ with exactly two objects $\Sigma$ and $\Sigma^{\prime}$, and $\sigma: \Sigma \rightarrow \Sigma^{\prime}$ as the only non-identity morphism. The sentence functor is given by $\operatorname{Sen}_{1}(\Sigma)=\left\{\varphi, \varphi^{\prime}\right\}, \operatorname{Sen}_{1}\left(\Sigma^{\prime}\right)=\left\{\varphi, \varphi^{\prime}, \psi\right\}$, with $\sigma$-translation preserving $\varphi$ and $\varphi^{\prime}$. Define $\mathcal{E}^{1}$ as the least entailment system for $\mathbf{S e n}_{1}$ such that $\psi \vdash_{\Sigma^{\prime}}^{1} \varphi, \varphi \vdash_{\Sigma}^{1} \varphi^{\prime}$.

Then consider the property-oriented semantics $\mathcal{T}^{\prime \prime}$ defined in Example 5.4. It is $\mathcal{E}^{1}$ sound (for the same reason as in Example 5.4), compositional and extensive. Moreover, for $S P_{b a d}$ defined as $\left\langle\Sigma^{\prime},\{\psi\}\right\rangle$ hide via $\sigma$, we have $\mathcal{T}^{\prime \prime}\left(S P_{b a d}\right.$ with $\left.\sigma\right)=\left\{\psi, \varphi, \varphi^{\prime}\right\}$, while $\mathcal{T}_{\mathcal{E}^{1}}\left(S P_{\text {bad }}\right.$ with $\left.\sigma\right)=\left\{\varphi, \varphi^{\prime}\right\}$.

As in Sect. 5, the counterexample semantics above had to be non-monotone:

Corollary 6.8. $\mathcal{T}_{\mathcal{E}}$ is at least as strong as any $\mathcal{E}$-sound, monotone and extensive propertyoriented semantics for structured specifications built from flat specifications using union, translation and hiding.

Proof. Let $\mathcal{T}$ be a $\mathcal{E}$-sound, monotone and extensive property-oriented semantics. Consider the institution $\mathbf{I N S}_{\mathcal{E}}$ where semantic entailment coincides with entailment in $\mathcal{E}$, as given by Prop. 6.2. Then $\mathcal{T}_{\mathcal{E}}$ coincides with $\mathcal{T}_{\mathbf{I N S}_{\mathcal{E}}}$, and $\mathcal{T}$ is sound in $\mathbf{I N S}_{\mathcal{E}}$ (as well as monotone and extensive). Consequently, $\mathcal{T}_{\mathcal{E}}$ is at least as strong as $\mathcal{T}$ by Cor. 5.6.

Clearly, the requirement that the semantics considered in the above corollary be extensive cannot be dropped here by Example 6.6, since the semantics $\mathcal{T}^{\prime}$ given there is monotone.

We conclude this section by comparing semantics in different entailment systems. Of course, if $\mathcal{E}$ is not at least as strong as another entailment system $\mathcal{E}^{\prime}$ used to give a semantics for specifications, we cannot expect $\mathcal{T}_{\mathcal{E}}$ to be at least as strong as this other semantics; typically this would not hold even for flat specifications. However:

Proposition 6.9. Consider two entailment systems for Sen : Sign $\rightarrow$ Set, $\mathcal{E}=\left\langle\vdash_{\Sigma}\right\rangle_{\Sigma \in|\operatorname{Sign}|}$ 
and $\mathcal{E}^{\prime}=\left\langle\vdash_{\Sigma}^{\prime}\right\rangle_{\Sigma \in|\operatorname{Sign}|}$. Suppose that $\mathcal{E}$ is at least as strong as $\mathcal{E}^{\prime}$, that is, for each signature $\Sigma \in|\operatorname{Sign}|, \vdash_{\Sigma}^{\prime} \subseteq \vdash_{\Sigma}$ (all $\mathcal{E}^{\prime}$-consequences of any set of sentences are also its $\mathcal{E}$-consequences). Then $\mathcal{T}_{\mathcal{E}}$ is at least as strong as $\mathcal{T}_{\mathcal{E}^{\prime}}$ for specifications built from flat specifications by union, translation and hiding: $\mathcal{T}_{\mathcal{E}^{\prime}}(S P) \subseteq \mathcal{T}_{\mathcal{E}}(S P)$ for all $S P \in$ Spec $^{U T H}$.

Proof. By easy induction on the structure of specifications.

Corollary 6.10. $\mathcal{T}_{\mathcal{E}}$ is at least as strong as any semantics for specifications built from flat specifications using union, translation and hiding that is compositional, extensive, $\mathcal{E}^{\prime}$-theory-oriented and $\mathcal{E}^{\prime}$-sound for some entailment system $\mathcal{E}^{\prime}$ such that $\mathcal{E}$ is at least as strong as $\mathcal{E}^{\prime}$.

Proof. By Cor. 6.5 and Prop. 6.9.

It may be considered somewhat unsatisfactory to require that the semantics we compare $\mathcal{T}_{\mathcal{E}}$ with is $\mathcal{E}^{\prime}$-sound, rather than just $\mathcal{E}$-sound. However, this cannot be weakened, since the counterexample semantics $\mathcal{T}^{\prime \prime}$ given in Example 6.7 is $\mathcal{E}^{\prime}$-theory-oriented for instance for the least entailment system $\mathcal{E}^{\prime}$ generated by $\psi \vdash_{\Sigma^{\prime}}^{\prime} \varphi$ (and any system that is still weaker than such $\left.\mathcal{E}^{\prime}\right)$.

However, as discussed at the end of Sect. 5, the perhaps unexpected requirements on the property-oriented semantics considered may be dropped if a stronger version of soundness and compositionality is assumed, that persist when the specification framework is extended.

We say that a property-oriented semantics $\mathcal{T}$ for specifications built using some specificationbuilding operations in the context of an entailment system $\mathcal{E}$ is $\mathcal{E}$-persistently sound and compositional if it is persistently sound and compositional in any institution INS for which $\mathcal{E}$ is sound.

Corollary 6.11. $\mathcal{T}_{\mathcal{E}}$ is at least as strong as any $\mathcal{E}$-persistently sound and compositional property-oriented semantics for structured specifications built from flat specifications using union, translation and hiding.

Proof. By Cor. 5.8, considering the institution $\mathbf{I N S}_{\mathcal{E}}$ where semantic entailment coincides with entailment in $\mathcal{E}$, as given by Prop. 6.2, and $\mathcal{T}_{\mathcal{E}}$ coincides with $\mathcal{T}_{\mathbf{I N S}_{\mathcal{E}}}$.

\section{Final remarks}

We studied property-oriented semantics for structured specifications in the context of an arbitrary institution, and then in the context of an arbitrary entailment system.

Considering specifications built from flat specifications using union, translation and hiding, we explained why the standard compositional property-oriented semantics given in Sect. 4 cannot be improved. On one hand, we sharpened the standard result (Borzyszkowski, 2002) that this semantics is complete in any exact institution with an appropriate interpolation property (cf. Thm. 4.4). On the other hand, we showed that it is at least as strong as any other sound, compositional, extensive theory-oriented semantics, as well as any other sound, monotone, extensive property-oriented semantics (cf. Cor. 5.2 and Cor. 5.6). These two results follow from more general theorems that state similar results 
for specifications built using an arbitrary collection of specification-building operations (cf. Thm. 5.1 and Thm. 5.5). We also give counterexamples that show that the unexpected and counter-intuitive requirements of extensiveness (the semantics considered must not "forget" about axioms in flat specifications ${ }^{5}$ ) and theory-orientedness (they take regard of consequences of the properties derived) cannot be dropped in general (cf. Examples 5.3 and 5.4). However, they are superfluous if we require a stronger form of soundness and compositionality, that persist when the specification formalism is extended by new specification constants with arbitrary sound semantics (cf. Thm. 5.7 and Cor. 5.8). It is worth noting that a similar effect may be achieved if instead of adding new specification constants we require that the property-oriented semantics for structured specifications translated by any institution comorphism (Meseguer, 1989; Tarlecki, 2000; Goguen and Roşu, 2002) extends in a sound and compositional way to structured specifications in the richer institution.

These results and counterexamples improve significantly on related results in (Sannella and Tarlecki, 2012). They carry over to the context of specifications built from flat specifications using union, translation and hiding in the context of an entailment system, see Sect. 6. The results also apply, mutatis mutandis, to any specification language that has at least the expressive power provided by these simple operations.

Although we discussed property-oriented semantics here, there is an intimate link between proof systems and property-oriented semantics which make the results immediately applicable to proof systems as well. For instance, the standard compositional propertyoriented semantics $\mathcal{T}_{\text {INS }}$ for structured specifications built from flat specifications using union, translation and hiding in an institution INS given in Sect. 4 may be presented using the following well-known proof system:

$$
\begin{gathered}
\overline{\langle\Sigma, \Phi\rangle \vdash \varphi} \quad \varphi \in \Phi \\
\frac{S P_{1} \vdash \varphi}{S P_{1} \cup S P_{2} \vdash \varphi} \quad \frac{S P_{2} \vdash \varphi}{S P_{1} \cup S P_{2} \vdash \varphi} \\
\frac{S P \vdash \varphi}{S P \text { with } \sigma \vdash \sigma(\varphi)} \quad \frac{S P \vdash \sigma(\varphi)}{S P \text { hide via } \sigma \vdash \varphi}
\end{gathered}
$$

together with the following rule to link consequences of specifications with semantic consequence in the underlying institution:

$$
\frac{S P \vdash \varphi \text { for each } \varphi \in \Phi \quad \Phi \models \psi}{S P \vdash \psi} \quad(=\text { closure })
$$

Clearly, for any specification $S P \in \operatorname{Spec}^{U T H}$ and $\operatorname{Sig}[S P]$-sentence $\varphi$, we have that $\varphi \in$ $\mathcal{T}_{\text {INS }}(S P)$ if and only if $S P \vdash \varphi$ can be derived in the above proof system. The standard compositional property-oriented semantics $\mathcal{T}_{\mathcal{E}}$ for structured specifications built from flat specifications using union, translation and hiding in an entailment system $\mathcal{E}$ given

\footnotetext{
${ }^{5}$ For this reason, extensiveness is called non-absent-mindedness in (Sannella and Tarlecki, 2012).
} 
in Sect. 6 may be presented by essentially the same proof system with the final rule $(\vDash$ closure) replaced by the following:

$$
\frac{S P \vdash \varphi \text { for each } \varphi \in \Phi \quad \Phi \vdash_{S i g[S P]} \psi}{S P \vdash \psi} \quad(\vdash \text { closure })
$$

In such a sense, any proof system for proving consequences of specifications generates a property-oriented semantics. (Note that this is different from proof systems for proving entailment between properties in the context of a structured specification, as studied in (Diaconescu, 2008, Sect. 14.2).)

On one hand then, the notions we introduced for property-oriented semantics, like soundness, completeness, closed-completeness, compositionality, monotonicity, etc., may be directly applied to proof systems. In particular, the proof system given by the rules above is sound, theory-oriented, monotone, compositional, extensive, flat-complete and closed-complete for the specification-building operations considered. The main results presented here for property-oriented semantics carry over to proof systems as well, and can be recast as establishing that the above proof system for consequences of structured specifications built from flat specifications using union, translation and hiding is the strongest compositional one possible. Improving on it requires compositionality to be sacrificed: a non-compositional proof system that is stronger may be given for instance by the following single rule, using Theorem 3.1:

$$
\frac{\Phi^{\prime} \vdash_{\Sigma^{\prime}} \sigma(\varphi)}{S P \vdash \varphi} \operatorname{nf}(S P)=\left\langle\Sigma^{\prime}, \Phi^{\prime}\right\rangle \text { hide via } \sigma
$$

Another non-compositional approach, which uses additional axioms and rules that are derived from the form of the specification in question, is (Hennicker et al., 1997).

On the other hand, we may want to study formats of proof systems that ensure desirable properties of the semantics they generate. For instance, if all the proof rules in a proof system derive consequences of structured specifications from consequences of their immediate constituents then the corresponding property-oriented semantics is compositional. Monotonicity follows if furthermore none of the proof rules involves "negative" premises. Finally, the property-oriented semantics given by a proof system is theory-oriented (resp. $\mathcal{E}$-theory-oriented) iff the rule $(\models$ closure) (resp. $(\vdash$ closure $))$ is admissible.

Acknowledgements. Our thanks to the anonymous reviewers for their perceptive comments, and to one of them for reminding us to explain the relationship to (Goguen and Roşu, 2004). This work has been partially supported by the Polish Ministry of Science and Higher Education, grant N206 493138 (AT).

\section{References}

Bergstra, J. A., Heering, J., and Klint, P. (1990). Module algebra. Journal of the Association for Computing Machinery, 37(2):335-372.

Bidoit, M. and Mosses, P. D., editors (2004). CAsL User Manual, Lecture Notes in Computer Science, Vol. 2900. Springer. See also http://www.informatik.uni-bremen.de/cofi/wiki/ index.php/CASL. 
Borzyszkowski, T. (2002). Logical systems for structured specifications. Theoretical Computer Science, 286(2):197-245.

Borzyszkowski, T. (2005). Generalized interpolation in first order logic. Fundamenta Informaticae, 66(3):199-219.

Burstall, R. M. and Goguen, J. A. (1977). Putting theories together to make specifications. In: Fifth International Joint Conference on Artificial Intelligence, pages 1045-1058, Boston.

Burstall, R. M. and Goguen, J. A. (1980). The semantics of Clear, a specification language. In: Bjørner, D., editor, Proceedings of the 1979 Copenhagen Winter School on Abstract Software Specification, Lecture Notes in Computer Science, Vol. 86, pages 292-332. Springer.

Burstall, R. M. and Goguen, J. A. (1981). An informal introduction to specifications using Clear. In: Boyer, R. S. and Moore, J. S., editors, The Correctness Problem in Computer Science, pages 185-213. Academic Press. Also in: Narain Gehani and Andrew D. McGettrick, editors, Software Specification Techniques. Addison-Wesley, 1986.

Chang, C.-C. and Keisler, H. J. (1990). Model Theory. North-Holland, third edition.

Diaconescu, R. (2008). Institution-Independent Model Theory. Birkhäuser.

Diaconescu, R., Goguen, J. A., and Stefaneas, P. (1993). Logical support for modularisation. In: Huet, G. and Plotkin, G., editors, Logical Environments, pages 83-130. Cambridge University Press.

Ehrig, H., Wagner, E. G., and Thatcher, J. W. (1983). Algebraic specifications with generating constraints. In: Proceedings of the 10th International Colloquium on Automata, Languages and Programming, Barcelona, Lecture Notes in Computer Science, Vol. 154, pages 188-202. Springer.

Goguen, J. A. and Burstall, R. M. (1992). Institutions: Abstract model theory for specification and programming. Journal of the Association for Computing Machinery, 39(1):95-146.

Goguen, J. A. and Roşu, G. (2002). Institution morphisms. Formal Aspects of Computing, 13(3-5):274-307.

Goguen, J. A. and Roşu, G. (2004). Composing hidden information modules over inclusive institutions. In: From Object-Orientation to Formal Methods. Essays in Memory of Ole-Johan Dahl, Lecture Notes in Computer Science, Vol. 2635, pages 96-123. Springer.

Harper, R., Sannella, D., and Tarlecki, A. (1994). Structured presentations and logic representations. Annals of Pure and Applied Logic, 67:113-160.

Hennicker, R., Wirsing, M., and Bidoit, M. (1997). Proof systems for structured specifications with observability operators. Theoretical Computer Science, 173(2):393-443.

MacQueen, D. and Sannella, D. (1985). Completeness of proof systems for equational specifications. IEEE Transactions on Software Engineering, SE-11(5):454-461.

Meseguer, J. (1989). General logics. In: Ebbinghaus, H.-D., editor, Logic Colloquium '87, Granada, pages 275-329. North-Holland.

Mossakowski, T., Autexier, S., and Hutter, D. (2006). Development graphs — proof management for structured specifications. Journal of Logic and Algebraic Programming, 67(1-2):114-145.

Mosses, P. D., editor (2004). CASL Reference Manual, Lecture Notes in Computer Science, Vol. 2960. Springer.

Rodenburg, P. H. (1991). A simple algebraic proof of the equational interpolation theorem. Algebra Universalis, 28:48-51.

Roşu, G. and Goguen, J. A. (2000). On equational Craig interpolation. Journal of Universal Computer Science, 6(1):194-200.

Sannella, D., Sokołowski, S., and Tarlecki, A. (1992). Toward formal development of programs from algebraic specifications: Parameterisation revisited. Acta Informatica, 29(8):689-736. 
Sannella, D. and Tarlecki, A. (1988). Specifications in an arbitrary institution. Information and Computation, 76(2-3):165-210.

Sannella, D. and Tarlecki, A. (2012). Foundations of Algebraic Specification and Formal Software Development. Monographs in Theoretical Computer Science. An EATCS Series. Springer.

Sannella, D. and Wirsing, M. (1983). A kernel language for algebraic specification and implementation. In: Karpinski, M., editor, Proceedings of the 1983 International Conference on Foundations of Computation Theory, Borgholm, Lecture Notes in Computer Science, Vol. 158, pages 413-427. Springer.

Tarlecki, A. (1986). Bits and pieces of the theory of institutions. In: Pitt, D. H., Abramsky, S., Poigné, A., and Rydeheard, D. E., editors, Proceedings of the Tutorial and Workshop on Category Theory and Computer Programming, Guildford, Lecture Notes in Computer Science, Vol. 240, pages 334-360. Springer.

Tarlecki, A. (2000). Towards heterogeneous specifications. In: Gabbay, D. and de Rijke, M., editors, Frontiers of Combining Systems 2, Studies in Logic and Computation, Vol. 7, pages 337-360. Research Studies Press.

Tarlecki, A. (2011). Some nuances of many-sorted universal algebra: A review. Bulletin of the European Association for Theoretical Computer Science, 104:89-111. 